\title{
Modelling Social Influence and Cultural Variation in Global Low-Carbon Vehicle Transitions
}

\author{
Pettifor, H.* \\ Tyndall Centre for Climate Change Research, University of East Anglia, Norwich NR4 7TJ, UK \\ h.pettifor@uea.ac.uk \\ $\underline{\mathrm{Tel}+441603592547}$ \\ Wilson, C. \\ Tyndall Centre for Climate Change Research, University of East Anglia, Norwich NR4 7TJ, UK \\ charlie.wilson@uea.ac.uk \\ McCollum, D. \\ Energy Program, International Institute for Applied Systems Analysis (IIASA), Laxenberg, Austria \\ mccollum@iiasa.ac.at \\ Edelenbosch, $\mathrm{O}$. \\ PBL Netherlands Environment Assessment Agency, Bilthoven, Netherlands \\ Oreane.edelenbosch@pbl.n
}

*corresponding author

\begin{abstract}
We present a unique and transparent approach for incorporating social influence effects into global integrated assessment models used to analyse climate change mitigation. We draw conceptually on Rogers (2003) diffusion of innovations, introducing heterogeneous and interconnected consumers who vary in their aversion to new technologies. Focussing on vehicle choice, we conduct novel empirical research to parameterise consumer risk aversion and how this is shaped by social and cultural influences. We find robust evidence for social influence effects, and variation between countries as a function of cultural differences. We then formulate an approach to modelling social influence which is implementable in both simulation and optimisation-type models. We use two global integrated assessment models (IMAGE and MESSAGE) to analyse four scenarios that introduce social influence and cultural differences between regions. These scenarios allow us to explore the interactions between consumer preferences and social influence. We find that incorporating social influence effects into global models accelerates the early deployment of electric vehicles and stimulates more widespread deployment across adopter groups. Incorporating cultural variation leads to significant differences in deployment between culturally divergent regions such as the USA and China. Our analysis significantly extends the ability of global integrated assessment models to provide policy-relevant analysis grounded in real world processes.
\end{abstract}

Highlights

- A formulation for adding social influences into global transport models is proposed

- This draws together strong conceptual thinking with robust empirical evidence

- Adding social influences speeds up the diffusion of alternative fuel vehicles

- And varied according to cultural differences between model countries/regions

Keywords

Social influence, behavioural realism, vehicle choice, AFV

This work was funded by European Union's Seventh Programme for research, technological development and demonstration under grant agreement number. 308329 


\section{Introduction}

Global integrated assessment models (IAMs) are widely used to evaluate the costs, potentials and consequences of different greenhouse gas emission trajectories. Representing the global energy and land use systems linked to the broader economy, they provide valuable insights into the medium and long term effects of socio-economic development, technological change and climate policy (Krey, 2014). Although modelling on this scale is inevitably stylised, global IAMs provide insights into the consequences of policy choices (Clarke et al., 2014). These insights are generated by exploring robust features of uncertain futures based on scenario assumptions.

Vehicle purchase is a technology adoption decision that will strongly influence future energy and emission outcomes (Girod et al., 2013). The transportation sector is responsible for around $23 \%$ of energy-related $\mathrm{CO}_{2}$ emissions. Despite innovations in low-carbon fuel technologies and policies to stimulate alternative fuel vehicle (AFV) adoption, transport emissions worldwide continue to grow faster than any other end-use sector. Around $80 \%$ of this increase is attributable to road vehicles (Sims et al., 2014). Vehicles are relatively short-lived capital assets and therefore vehicle fleet turnover opens up opportunity to adopt new types of technologies. However IAM projections suggest there are immense decarbonisation challenges within road transport with slow transition from conventional vehicles (CVs) to electric, biofuel, or hydrogen-powered AFVs. Strong climate policy as well as sectoral transport policies are needed to drive the transition from conventional to alternative fuel vehicles (Creutzig et al., 2015).

IAMs represent vehicle choice and transitions between fuels and vehicle technologies. With their necessary levels of aggregation, due to their global scope and long time frames, IAMs have a simplified representation of consumer choice. Consumers behave as individual rational agents making discrete choices between alternative vehicle technologies based on their preferences for cost and efficiency attributes within income constraints (McCollum et al., 2016; Mercure et al., 2016). As vehicles are expensive capital goods, income availability is a major source of heterogeneity between consumers and rises in income are strongly correlated with consumer's willingness to pay for more powerful vehicles (Mercure and Lam, 2015). In reality, consumer choices are based on a range of other non-financial and non-energy related criteria. There are many types of non-financial consumer preferences, including current use patterns (people buy what they know), aesthetics (e.g., style, comfort, convenience) and performance (e.g., speed, reliability, safety) (Avineri, 2012; Mattauch et al., 2015).Modelled consumer choices reflect a response to changing technology and resource costs rather than other more complex and non-financial decision processes (Arneth et al., 2014; Mattauch et al., 2015). This limits the models' usefulness to policy makers if they cannot realistically simulate the effect on behaviour of different policy instruments (Rivers and Jaccard, 2005).

We focus on a particularly important aspect of consumer choice: social influence. Social influence is the process by which consumer attitudes and behaviours towards an innovation are shaped by interactions with others. Consumers demonstrate new technology and are particularly influential if they are perceived by others to have expert knowledge or experience (Friedkin and Johnsen, 1990; Lavine and Latané, 1996).

Empirical research shows that vehicle purchases are strongly socially and culturally determined. Vehicle purchases do not only satisfy financial criteria but also contribute towards self-identity, convey status to others, and enable group membership (Axsen et al., 2013; Schuitema et al., 2013). People rely on the opinion and behaviour of others around them to communicate not only the acceptability of owning particular vehicles but also to signal reliability and quality which is particularly relevant for vehicles with new fuel or body types (Adjemian et al., 2010; Gaker et al., 
2010; Heutel and Muehlegger, 2010; Wiedmann et al., 2011). People especially rely on the opinion and behaviour of those within close social networks including friends, family, neighbours, and work colleagues (Adjemian et al., 2010; Aini et al., 2013; Axsen et al., 2013; Grinblatt et al., 2008; McShane et al., 2012). These social influences apply equally to purchases of conventional vehicles (CVs) and alternative fuel vehicles (AFVs) (Pettifor et al., 2017)

Consumer choice is also shaped by broader social beliefs and value systems that prevail within a given culture. This creates distinctions between countries and regions in terms of consumer behaviour and preferences for products (Bauernschuster and Falck, 2015; Caragliu and Nijkamp, 2016; de Mooij and Hofstede, 2002; Dwyer et al., 2005). Dwyer et al. (2005) show countries vary in terms of people's receptiveness to influences from social trends in consumption ("keeping up with the Jones"). Differences in social influence between countries are confirmed in a meta-analysis of studies examining social influence on vehicle purchases (Pettifor et al., 2017)

Global IAMs used to analyse policy effectiveness need to capture the social and cultural influences that shape behaviour (Mercure et al., 2016). The effectiveness of policies to reduce emissions from private vehicles depends on incentivising consumer choices towards alternative fuel vehicles (AFVs). These consumer choices are not only discrete cost-optimising decisions but also socially and culturally determined.

The aim of this paper is to use a clear conceptualisation of social influence backed by strong empirical evidence to improve the behavioural realism of global IAMs used in climate policy analysis. Our aim is not to improve policy simulation but to fill a major gap in the literature between contextualised empirical studies and stylised global modelling tools. We focus on introducing social influences on consumer preferences for new vehicles: both CVs and AFVs. We develop and implement a novel model formulation for social influence which uses empirical data from primary studies and introduces cultural variation between model regions. We implement this formulation in two global IAMs with different designs. We then run scenarios to test the effect of adding social influences. Our approach works within the constraints of cost-optimisation modelling in which consumers have perfect information about available alternatives (Trutnevyte, 2016). However we introduce 'intangible' costs to reflect consumers' non-financial preferences including towards risk and uncertainty. The result is a closer approximation between consumer choice and the models' necessarily stylised representations of decision making. Our efforts build on other research whose aim is to improve cost-optimising models' ability to represent real-world processes (Ekholm et al., 2010; Keppo and Strubegger, 2010; McCollum et al., 2016).

Table 1 - Summary of abbreviations used in this paper.

\begin{tabular}{|c|c|c|c|c|}
\hline & Abb & viati & & Definitions \\
\hline \multirow{3}{*}{ models } & \multicolumn{3}{|c|}{ IAM } & integrated assessment model \\
\hline & .. & \multicolumn{2}{|c|}{ MESSAGE } & a global inter-temporal optimisation model \\
\hline & .. & \multicolumn{2}{|c|}{ IMAGE } & a global dynamic recursive simulation model \\
\hline \multirow{6}{*}{ vehicle types } & \multicolumn{3}{|l|}{$\mathrm{CV}$} & conventional vehicle \\
\hline & \multicolumn{3}{|l|}{ AFV } & alternative fuel vehicles \\
\hline & .. & \multicolumn{2}{|l|}{ EV } & electric vehicles \\
\hline & &.. & BEV & battery electric vehicles \\
\hline & & & PHEV & plug-in hybrid electric vehicles \\
\hline & .. & Otl & AFV & other hybrid vehicles, biofuels, hydrogen fuel cells \\
\hline \multirow{5}{*}{$\begin{array}{l}\text { heterogeneous } \\
\text { adopters } \\
\text { (based on } \\
\text { Rogers 2003) }\end{array}$} & \multicolumn{3}{|l|}{ IN } & innovators \\
\hline & \multicolumn{3}{|l|}{ EA } & early adopters \\
\hline & \multicolumn{3}{|l|}{ EM } & early majority \\
\hline & \multicolumn{3}{|l|}{ LM } & late majority \\
\hline & \multicolumn{3}{|l|}{ LG } & laggards \\
\hline
\end{tabular}




\section{Literature Review}

2.1 Diffusion as social influence among heterogeneous adopters

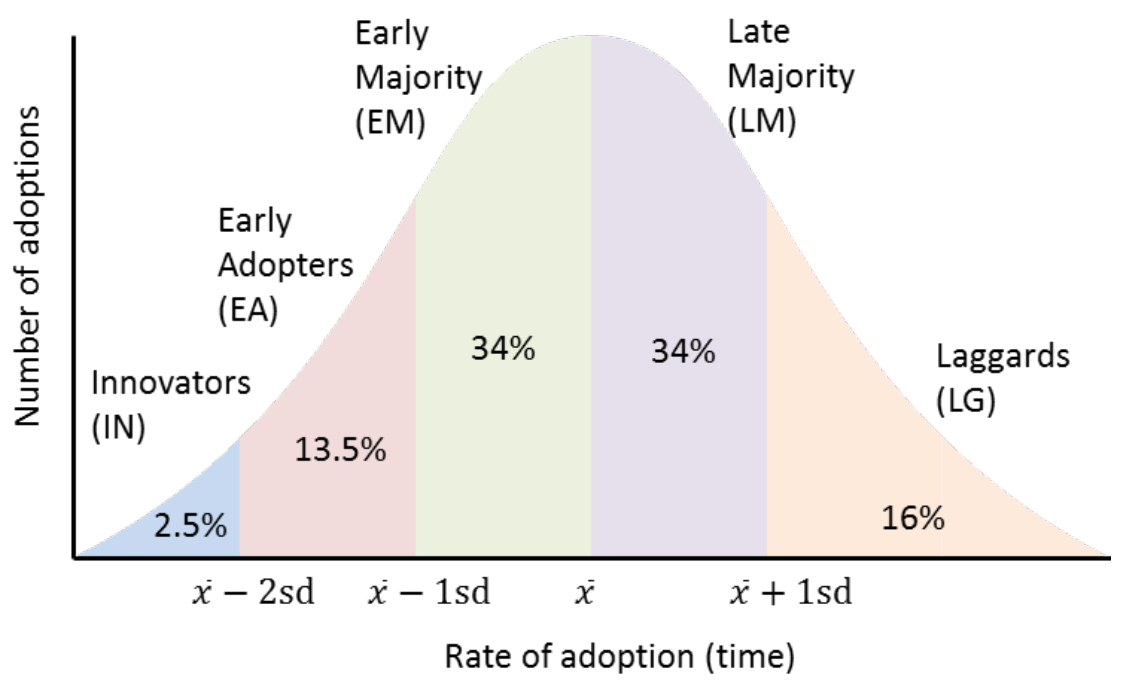

Figure 1 - Identification of adopter groups by Rogers (2003)

Consumer choices of most relevance to IAMs relate to technology adoption and subsequent use. The dominant theoretical framework used in the analysis of technology adoption is Rogers' diffusion of innovations (Rogers, 2003). This describes the process by which innovations diffuse as information on their attributes, costs and benefits is communicated among members of a social system and so reduces uncertainty and perceived risks of adoption. Roger's diffusion of innovations theory is built on empirical evidence from many studies of adoption in the USA between 1948 and 1973 all which show the classic bell shaped diffusion curve. A more recent review of diffusion of innovation theory and its modelling approaches confirms its continued relevance and importance (Meade and Islam, 2006). Characteristic within Rogers' frequency distribution is the use of point estimates (the mean and standard deviation) to divide the distribution of adoption propensities into 'ideal types' referred to as adopter groups (Figure 1). Innovations appeal initially to innovators (IN) who seek novel performance attributes and have a high risk tolerance. Based on a synthesis of empirical research, Rogers (2003) generalises the IN group to consist of the first $2.5 \%$ of potential adopters. Assuming adoption propensities are normally distributed, this IN group occupies the area two or more standard deviations (sd) below the mean (Figure 1). Diffusion has two key elements relevant here: (1) social influence; (2) heterogeneous adoption propensities.

There are many types of social influence. Examples include descriptive and injunctive norms (Cialdini et al., 1991), imitation effects (also herding behaviour and bandwagon effects) (Bass, 1969) localised neighbourhood effects, and status seeking (Griskevicius et al., 2010). In a synthesis of 72 empirical studies specifically examining social influence effects on vehicle choice, (Pettifor et al., 2017) identify three main types of social influence: interpersonal communication, neighbourhood effect, and social norms (see Supplementary Information A).

All these types of social influence occur because of variation in the adoption propensity and risk aversion within a population of potential adopters. Characteristic within Rogers' frequency distribution is the use of point estimates (the mean and standard deviation) to divide the distribution of adoption propensities into 'ideal types' referred to as adopter groups (Figure 1). Innovations appeal initially to innovators (IN) who seek novel performance attributes and have a high risk tolerance. Based on a synthesis of empirical research, Rogers (2003) generalises the IN 
group to consist of the first $2.5 \%$ of potential adopters. Assuming adoption propensities are normally distributed, this IN group occupies the area two or more standard deviations (sd) below the mean (Figure 1). Early adopters (EA) are more risk averse than the IN group, but play an important role in diffusion processes as 'opinion leaders' to whom others look for information and experience. The early majority (EM) are the remaining adopter group with adoption propensities higher than the mean. The EA and EM groups occupy $13.5 \%$ and $34 \%$ of the population of potential adopters respectively. The final two groups of late majority (LM) and laggards (LG) have adoption propensities lower than the mean, representing respectively $34 \%$ and $16 \%$ of the adopter population. The LG group is particularly averse to risk and uncertainty, resistant to the influence of change agents (who try to guide their opinions), and so wait to adopt until an innovation is tried, tested, widely diffused, and so normalised (Figure 1).

\subsection{Modelling vehicle choices with non-financial preferences}

Discrete choice experiments are commonly used to measure propensity to purchase a particular vehicle type (Ahn et al., 2008; Axsen et al., 2009; Batley et al., 2004; Bunch et al., 1993). This method frames individuals as rational actors making deliberative choices between a given set of alternatives by weighing up the relative importance of both financial and non-financial attributes. Discrete choice experiments are particularly useful for testing responses to new technologies or new concepts with which people are otherwise unfamiliar or uncertain about, and for which market data does not yet exist. Derived within a random utility framework, decision makers are assumed to utility maximize, that is, they choose the product that provides them with the highest utility. In choice models for AFVs, for example, common attributes against which utility is measured are price, operating cost, $\mathrm{CO}_{2}$ emissions, engine power, range and refuelling availability (Achtnicht et al., 2012; Hackbarth and Madlener, 2013; Lebeau et al., 2012; Ziegler, 2012). The relative importance of non-financial preferences can be monetised in willingness to pay ratios (WTP) which provide further insight into the trade-offs people are willing to make at given price and attribute levels. As an example, one study found a consumer would be willing to pay on average up to US\$7,500 extra for an electric vehicle that could travel 100 miles further on a single charge (Dimitropoulos et al., 2013).

Vehicle choice and transport sector models have incorporated both financial and non-financial preferences in their representation of consumer choice (Brand et al., 2012; McCollum et al., 2016). One example is the MA3T vehicle choice model which has been developed for the USA by researchers at Oak Ridge National Laboratory (Lin et al., 2013; Lin and Greene, 2009). MA3T uses a nested multinomial logit discrete choice formulation and incorporates non-financial or 'intangible costs' for perceived risk, range anxiety, model availability, refuelling infrastructure, acceleration, and towing capability. Social influence effects can also be represented by a decline in perceived risk among majority consumers as a vehicle type becomes more mainstream.

\subsection{Cultural variation in consumer choice}

Empirical research shows there are significant cultural differences between countries which moderate technology purchase behaviour. By culture we refer to differences between prevailing belief and value systems (Hofstede, 2001). Hofstede (2001) cultural dimensions are a widely-used and standardised measure of culture, and have explained cultural differences in the purchasing behaviour of TVs, DVD players, cameras, washing machines, block buster movies and vehicles (de Mooij and Hofstede, 2002; Dwyer et al., 2005; Griffith et al., 2014; van Everdingen et al., 2009). For example, de Mooij and Hofstede (2002) compared the diffusion of vehicles across Europe, finding cultural differences accounted for different preferences for size and power of vehicles, design features and propensity to purchase new vehicles. 
Social influences on vehicle choice are also culturally mediated (Pettifor et al., 2017). They are stronger in more 'normative' countries such as USA where people are particularly receptive to social trends in consumption, have a greater focus on status obligations and material acquisition. In contrast social influences are weaker in more 'pragmatic' countries such as China where there is greater emphasis on longer term gain and willingness to adapt to changing social conditions (Dwyer et al., 2005; Minkov and Hofstede, 2012). Global surveys such as the World Values Survey provide quantified measures of cultural difference which inform comparative scales such as Hofstede's cultural dimensions (Hofstede, 2001). Scales range from 0 to 100 and measure culture through specific dimensions (see Supplementary Information C).

\subsection{Challenges for global IAMs in modelling vehicle choice}

Global IAMs tend to make three important simplifications in representing vehicle choice. First, they do not explicitly represent adopter heterogeneity (Mercure et al., 2016) but select technologies which have lowest lifecycle costs, avoiding 'winner takes all' behaviour by either constraining the rate at which new technologies can diffuse (optimisation models) or by ensuring market heterogeneity through the use of a logit-type function to assign market shares (recursive dynamic models). In both cases, there is a single decision function which results in a heterogeneous market outcome. This means social influence processes that drive diffusion are difficult to model, as these rely on varying adoption propensities among a population of adopters. Second, global IAMs assume the preferred alternative minimises levelised costs, accounting for both upfront costs and discounted future fuel and operating costs over the lifetime of the vehicle (Mattauch et al., 2015). This means the influence of non-financial preferences on vehicle choices cannot be modelled. Third, global IAMs assume stylised differences between countries, using differences in income or development levels to derive regionally-specific model parameterisations (Riahi et al., 2012). This means that cultural influences on vehicle choices cannot be modelled.

By omitting important real-world phenomena including adopter heterogeneity, social influence, nonfinancial preferences, and cultural variation, global IAMs are limited both in their behavioural realism and in their ability to inform policy (Creutzig et al., 2015). These limitations define a current frontier in global modelling analysis of climate change mitigation.

\section{Modelling Social Influence in Global IAMs}

\subsection{Model Formulation}

We develop a novel model formulation for incorporating social influences into global IAMs. We draw conceptually on Rogers (2003) to identify risk averse consumers (EM, LM, LG) who become more receptive to new technology as they observe the behaviour of early adopters (EA, incorporating IN). The underlying premise of our model formulation is that individual propensity to adopt a novel vehicle type is significantly affected by social influence. Specifically, the adoption experience generated by risk-tolerant early adopters reduces the risks perceived by more risk-averse later adopters. Our model formulation therefore improves the behavioural realism of global IAMs by introducing heterogeneous adopter groups, risk premiums as monetised attraction or aversion to AFVs, and social influence effects creating interdependence between early and later adopters.

1. Adopter groups. Consumer heterogeneity is introduced through four adopter groups. These groups are distinguished from each other by risk aversion and market share. The early adopters (EA) represent $16 \%$ of the total market, early majority (EM) $34 \%$, late majority (LM) $34 \%$ and laggards (LG) 16\%. (We combine the innovators and early adopters into a single group). 
2. Risk premium. Monetised risk aversion is introduced as an additional element in the levelised cost calculation which represents vehicle choice within the model. Initial risk premium values (US\$) are distinguished between the adopter groups (Table 2). The EA group, being less risk averse, have a negative risk premium whereas the EM, LM and LG groups have a positive risk premium. The risk premium values are estimated from a synthesis of discrete choice studies measuring stated preferences for AFVs which are captured in addition to preferences for specific vehicle attributes such as financial costs and range (see Supplementary Information B). We use all available willingness to pay ratios for AFVs in the literature, and assume they are normally distributed to calculate mean risk premium $(\bar{x} R P)$ and standard deviation risk premium $(\bar{\sigma} R P)$. These are then used to calculate initial risk premium values for EA, EM, LM, and LG using Rogers (2003) adoption propensity curve (Figure 1).

Table 2 - Initial risk premium values for each adopter group

\begin{tabular}{|l|l|l|l|l|}
\hline Adopter group & EA & EM & LM & LG \\
\hline Population share & $16 \%$ & $34 \%$ & $34 \%$ & $16 \%$ \\
\hline Initial risk premium (US\$) & $-\$ 3,533$ & $+\$ 2,637$ & $+\$ 6,313$ & $+\$ 13,665$ \\
\hline
\end{tabular}

3. Social influence effect. Propensity to purchase an AFV increases with evidence that others have adopted. To capture these effects in the model we introduce a linear function by which risk premium declines as market share grows. This approach assumes that the experiences of AFV adopters are 'communicated' through social influence processes to non-adopters whose perceived uncertainties towards AFVs are therefore reduced. Market share is thus a simple proxy for social influence effect. The strength of this relationship is derived from a metaanalysis. Of 43 studies identified through systematic literature review as measuring social influence effects on vehicle choice (conventional vehicles as well as AFVs), 29 studies were initially considered suitable for meta-analysis as they reported quantitative measures of social influence. However, six studies were excluded because they did not report specific effect sizes for social influence. A further two studies were excluded because they report outcome statistics that are not comparable to other studies (e.g., paired t-tests, rankings). Meta-analysis involves the identification of a comparable metric for each study, and the weighting of relative effect size according to sample size. The comparable metric we identify between studies is the correlation coefficient ( $r$ ) since all studies report either betas, log odds (probits), or chi-square statistics.

Figure 2 displays a forest plot with effect sizes for each study, the overall average effect size (0.241) and accompanying statistics from a random effects meta-analysis model. All individual effect sizes are positive and all but two are significant at $|p| \leq 0.05$. 


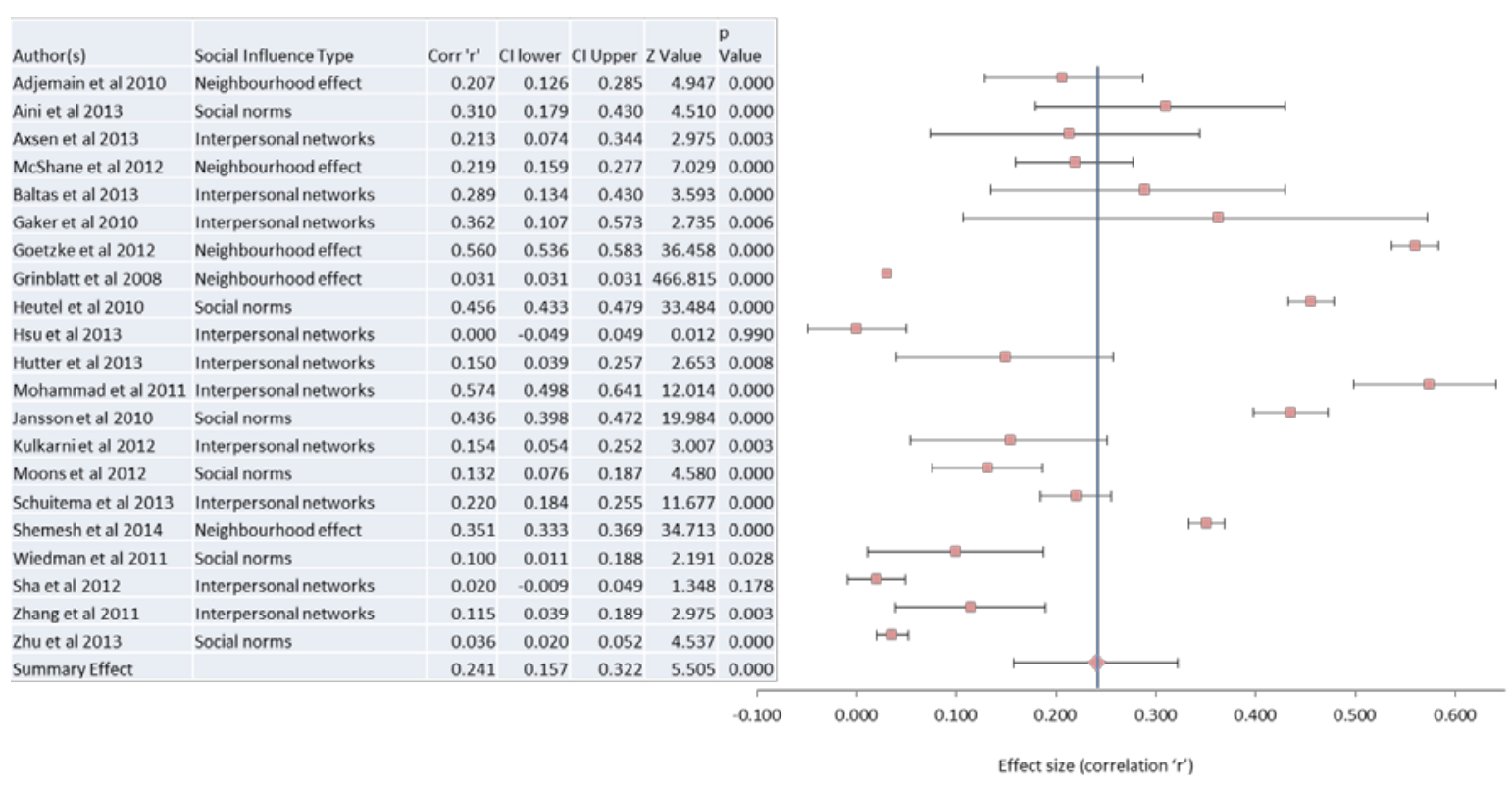

Figure 2 - Forest Plot of Social Influence Effect Sizes

Our meta-analysis of the 21 studies revealed an average effect size measured by the correlation coefficient ' $r$ ' of $0.241(95 \% \mathrm{Cl}[0.157,0.322], Z=5.505,|p| \leq 0.000)$. This is shown by the vertical line in Figure 2 and is considered to be a small to medium effect in meta-analysis (Cohen, 1992). For every one standard deviation increase in social influence the propensity of vehicle adoption increases by 0.241 standard deviations (see Supplementary Information A for additional information and Pettifor et al. (2017)for a full description of the meta-analysis). This effect of social influence on vehicle choices is small to moderate but significant. Importantly, it provides a robust and transparent empirical basis for the risk premium decline functions. These are specific to vehicle types aggregated into conventional vehicles (CV), electric vehicles (BEV and PHEV), and all other AFV types (Table 1, and Supplementary Information F). Taking BEVs as an example, an increasing market share for BEVs reduces the perceived risks with BEVs, but not other vehicle types; conversely, increasing adoption experience with other AFVs does not reduce the risk premiums for BEVs.

4. Cultural Variation. Social influence effects are not globally homogeneous. The meta-analysis returned a significant average effect size of beta $=0.241(95 \% \mathrm{Cl}[0.157,0.322], Z=5.505$, $|p| \leq 0.000)$. This average effect size was based on all studies and countries. However, further testing found that the average effect size was moderated by a country's cultural values, measured by a widely-used scale from 'pragmatic' to 'normative' (Minkov and Hofstede, 2012). This differentiation between countries in size of social influence effect was achieved through plotting the linear association between scores on the pragmatic versus normative scale and predicted social influence effect for the 11 countries sampled in the meta-analysis. The resultant linear equation $y=-0.428 x+0.4497$ (where $y=$ social influence effect size and $x=$ score on pragmatic versus normative scale $\left(R^{2}=0.82\right)$ ) was used to make further out of sample predictions to regionalise the social influence effect. Specifically, the strength of social influence varies with countries' position on the pragmatic/normative scale which measures cultural adaptation to different types of external influence (Hofstede, 2001). We use this relationship to introduce regionalised social influence effects (see Supplementary Information C). As examples, the USA is a more normative culture and more receptive to social influence effects (effect size 
increased from 0.241 to 0.368 ). Conversely, China is a more pragmatic culture with weaker social influences (effect size reduced from 0.241 to 0.108 ).

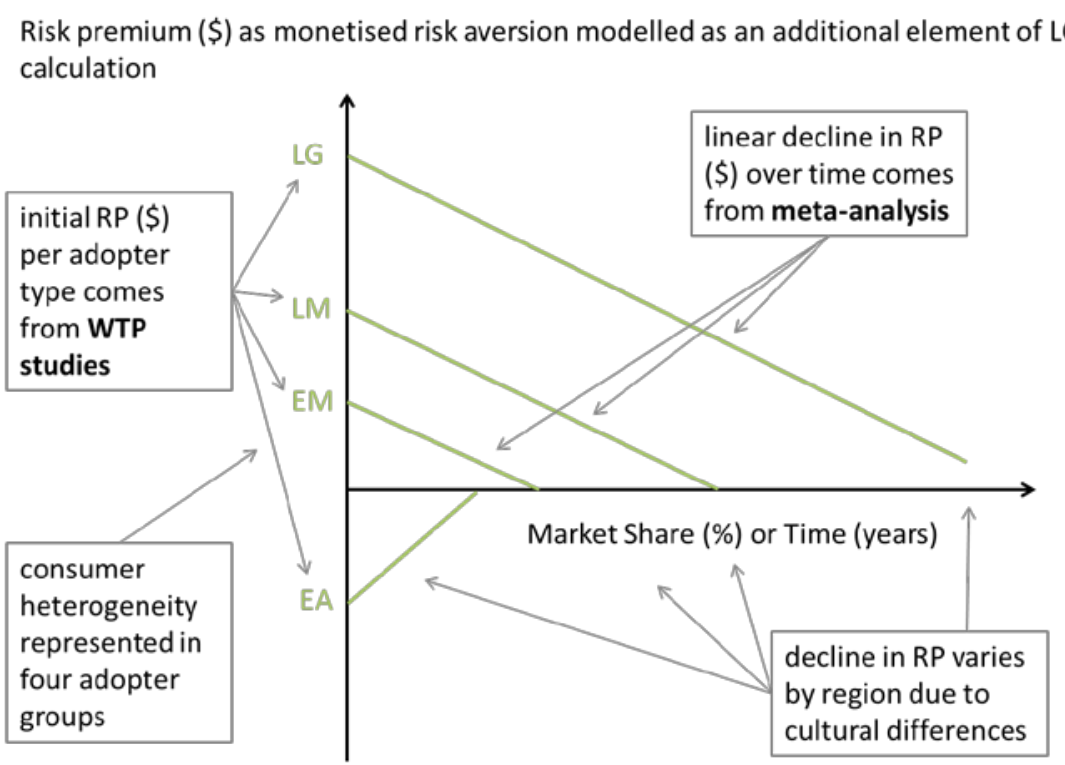

Figure 3 - Model formulation for incorporating social influence in global IAMs

Figure 3 illustrates our broad approach. In the endogenous model formulation the ' $x$ ' axis is measured in standard deviations of market share, for the exogenous model formulation this axis becomes time measured in standard deviations (years).

\subsection{Implementation of model formulation in global IAMs}

To implement this formulation we express social influence in terms of changes in vehicle market share $(\triangle M K)$ measured in standard deviation increments $(\bar{\sigma} M K)$. The effect of social influence on the increasing adoption propensity for AFVs is then expressed in terms of changes in risk premium ( $\triangle$ $R P)$ which is formally measured in standard deviation increments $(\bar{\sigma} R P)$. In other words, our model formulation assumes that social influence increases the adoption propensity of later adopters by reducing their risk aversion.

Our model formulation is versatile and can be adapted for global IAMs with different designs and solution algorithms. We demonstrate this by developing an endogenous formulation in which the social influence relationship is incorporated within the existing IAM structure. In this more direct interpretation of Rogers (2003) we simulate the interdependency between groups such that an increase in market share in the EA group endogenously results in a decline in risk premium in the EM, LM and LG groups.

We also develop an exogenous formulation in which the social influence relationship is calculated externally so that declining risk premiums are fed into the model as input assumptions. This exogenous formulation is necessary for inter-temporal optimisation IAMs which assume perfect foresight (e.g., vehicle choices are based on perfect information about all future technology and fuel costs). Because these models would 'see' all future benefits (or costs) of social influence on AFV purchases, they might select a costly early adoption of a new vehicle because of its long-term benefits in reducing costs for late adopters. This would be inconsistent with our efforts to improve the models' behavioural realism. The exogenous formulation therefore provides a workaround by iterating between a base model run without social influence effect (providing changes in market share) and the risk premium decline functions which feed social influence effects back into the model. Although this approach does not establish a true endogenous link between adopter types we 
include it to demonstrate not only the versatility of the approach by using a work-around solution but also to test whether this work-around solution produces markedly different results.

Model formulations are developed in IMAGE and MESSAGE. IMAGE is a global recursive-dynamic simulation model of interactions between natural and human systems. The system state in every future year running up to 2100 depends entirely on the previous year system state, based on a single set of deterministic algorithms (Stehfest et al., 2014). IMAGE includes a process model to describe the energy system, in which transport is an important energy consuming sector. Passenger and freight transport demand per mode type are modelled based on travel cost and mode speeds which are related to income and travel time. In each transport mode as is also the case for the light duty vehicle mode, a variety of vehicle types are represented, and vehicle shares are distributed through a multinomial logit equation (Girod et al., 2013).

MESSAGE is an inter-temporal optimization model combining a global (multi-region, multi sector) systems engineering model with an aggregated macro-economic model and a simple climate model, all within a consistent, inter-linked framework (McCollum et al., 2016). The version of the model we use here is MESSAGE-Transport, which contains a detailed transport module representing the light duty vehicle fleet and each of the other transport modes (McCollum et al., 2016).

IMAGE and MESSAGE both distinguish over 10 vehicle types which are available at varying cost levels and with varying performance characteristics (both of which can be improved through learning effects). In our model formulations, these vehicle fleet characteristics are common to all consumer groups. However, we also introduce varying 'intangible' cost terms as a measure of risk aversion and nonfinancial preferences. (An alternative approach is to use variable discount rates as a proxy for perceived risks or income constraints; we do not do this here).

\subsection{Endogenous formulation (IMAGE)}

For simulation-type models like IMAGE, change in risk premium $(\triangle R P)$ can be modelled as a direct function of changing market share $(\triangle M K)$. Adopters become less risk averse as evidence increases that others have adopted a new vehicle type. To divide market share growth into standard deviations $(\bar{\sigma} M K)$ we use (Rogers, 2003) adoption propensity curve assuming a normal distribution (Figure 4). This results in estimates of $2.5 \%$ market share $=2 \bar{\sigma} M K, 16 \%$ market share $=3 \bar{\sigma} M K, 50 \%$ market share $=4 \bar{\sigma} M K$ and so on (see Figure 3 ). These \% values do not scale linearly reflecting varying adoption propensities between adopter groups, consistent with (Rogers, 2003).

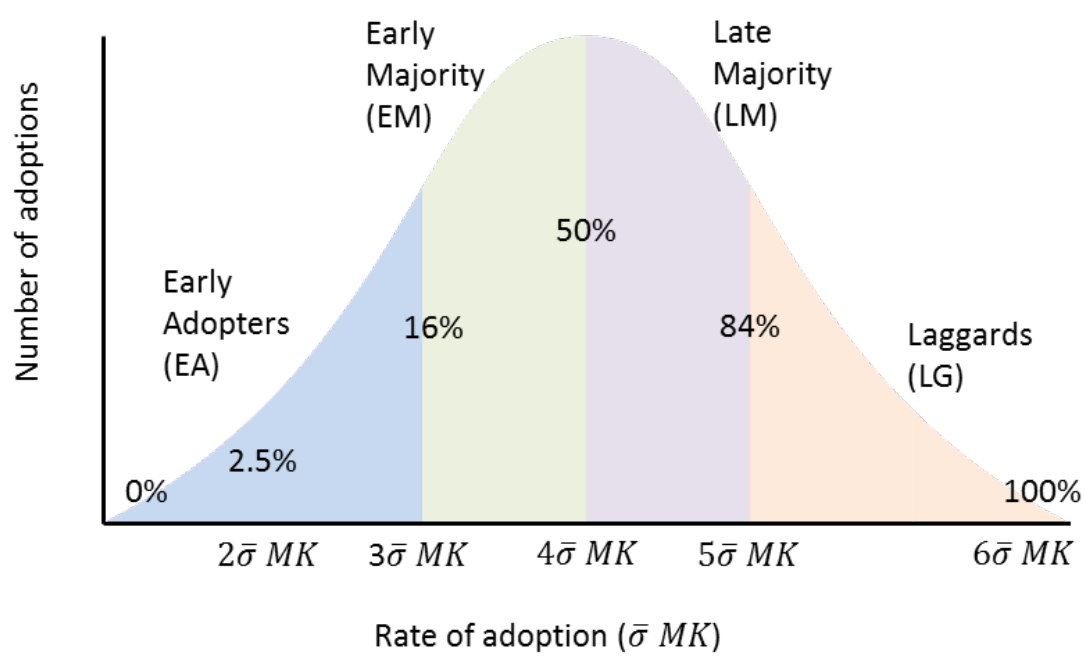

Figure 4 - Calculation of $\bar{\sigma} M K$ using (Rogers, 2003) adoption propensity curve 
Risk premium decline is then calculated in accordance with the basic meta-analytic result: for every one standard deviation increase in market share there is an equivalent 0.241 standard deviation decrease in risk premium $(+1 \bar{\sigma} M K=0.241 * \bar{\sigma} R P)$. We take $\bar{\sigma} R P$ from the synthesis of discrete choice studies (Supplementary Information B). The model interpolates between 0, 2.5\%, 16\%, 50\%, $84 \%$ and $100 \%$ market share, calculating the appropriate decline rate in risk premium relative to market share. This produces a stepwise linear decline function which over time resembles the ' $S$ ' shaped adopter curve (see Supplementary Information D).

\subsection{Exogenous formulation (MESSAGE)}

For optimisation-type models like MESSAGE, the relationship between changing risk premium $(\triangle R P)$ and changing market share $(\triangle M K)$ is implemented outside the model in an iteration between model runs with and without social influence effects. In this manner we are able to capture the basic relationship between time and market share, and use the risk premium decline function to calculate exogenously the risk premiums at each time step. This is done in accordance with the meta-analytic result $(+1 \bar{\sigma} M K=0.241 * \bar{\sigma} R P)$ and results in hardcoded risk premiums which can be fed back into the model as additional cost terms (see Supplementary Information E).

\subsection{Social influence scenarios}

Four scenarios are run to fully explore the consequences of adding social influence effects on vehicle choice into the global IAMs. All four scenarios are run using both endogenous and exogenous model formulations in IMAGE and MESSAGE respectively. The four scenarios are summarised in Table 3. All four scenarios include climate policy in the form of economy-wide carbon pricing (see next section).

Table 3 - Social influence scenarios

\begin{tabular}{|c|c|c|c|c|}
\hline & NoSI & GlobalSI & RegionaISI & StrongSI \\
\hline Description & $\begin{array}{l}\text { Counterfactual - no } \\
\text { social influence }\end{array}$ & $\begin{array}{l}\text { Global average social } \\
\text { influence accounting } \\
\text { for interdependencies } \\
\text { between } \\
\text { heterogeneous } \\
\text { adopter groups }\end{array}$ & $\begin{array}{l}\text { Regionalised social } \\
\text { influence accounting } \\
\text { for cultural variation } \\
\text { (as well as adopter } \\
\text { heterogeneity) }\end{array}$ & $\begin{array}{l}\text { Strong global average } \\
\text { social influence }\end{array}$ \\
\hline Social Influence & $\begin{array}{l}\text { No social influence } \\
\text { effect }\end{array}$ & $\begin{array}{l}\text { Global average social } \\
\text { influence effect }\end{array}$ & $\begin{array}{l}\text { Regionalised social } \\
\text { influence effect }\end{array}$ & $\begin{array}{l}\text { Upper bound of } \\
\text { global average social } \\
\text { influence effect }\end{array}$ \\
\hline Climate Policy & $\begin{array}{l}\text { Global carbon price of } \\
110 \$ / \mathrm{tCO}_{2} \text { from } 2020\end{array}$ & $\begin{array}{l}\text { Global carbon price of } \\
110 \$ / \mathrm{tCO}_{2} \text { from } 2020\end{array}$ & $\begin{array}{l}\text { Global carbon price of } \\
110 \$ / \mathrm{tCO}_{2} \text { from } 2020\end{array}$ & $\begin{array}{l}\text { Global carbon price of } \\
110 \$ / \mathrm{tCO}_{2} \text { from } 2020\end{array}$ \\
\hline
\end{tabular}

The NoSI scenario establishes model dynamics without social influence. Non-financial preferences related to consumer behaviour are captured by the initial risk premium values; however, these values do not decline over time nor as a function of market share. The NoSI scenario thus establishes a counterfactual reference point as in the real world consumers' risk aversion is shaped by market activity.

The GlobalSI scenario incorporates a global average social influence effect to assess the impact of social influence relative to the NoSI scenario. The RegionalSI scenario examines the impact of cultural variation in social influence by replacing the global average social influence effect with a regionalised social influence effect. 
The StrongSI scenario incorporates strong social influence based on the upper bound of the observed range of effect sizes within the meta-analysis. This is included as a sensitivity scenario to examine the global impact of increased social influence effect size.

\subsection{Cost determinants of AFV deployment and the role of climate policy}

In a global IAM - as in the real world - social influence has a secondary effect on vehicle choices. In other words, the risk premiums we introduce act as an additional influence on vehicle choices which are still predominantly characterised by financial attributes including upfront costs and fuel costs. The relative attractiveness of different vehicles on these cost attributes is determined endogenously by model processes. Upfront costs of specific vehicle types may change (e.g., through learning as a function of experience); fuel costs may change (e.g., through resource scarcity and trade, or through climate policy). For AFVs to become competitive, their levelised cost, taking both upfront costs and discounted fuel costs into account, needs to come down towards that of CVs. Stringent climate policy puts upwards pressure on the fuel costs of CVs, and so helps AFV competitiveness. This is general to all adopter groups. As AFVs approach CVs in levelised cost terms, the secondary effect of social influence may then potentially play a more defining role. This should be more marked initially in early adopters, and then on through the later adopting groups as their risk aversion declines.

We can already observe early adoption of AFVs in lead markets like Norway and California, thanks to dedicated AFV-supporting policies (Lipsey et al., 2005; Nilsson and Nykvist, 2016). However, global IAMs do not project significant market share growth of AFVs in the near-to-medium term as they are not sufficiently competitive with CVs. Global IAMs solve for cost-optimal mitigation pathways, and there tend to be lower cost emission reduction opportunities in the electricity and buildings sectors (Clarke et al., 2014).

We are interested in both near-to-medium term social influence processes which are already observable in the market for AFVs and interactions between social influence and climate policy in potentially accelerating low-carbon vehicle transitions. Consequently, all our social influence scenarios are set in the context of climate change mitigation and assume stringent climate policy to provide a strong price incentive to decarbonize the transport sector. Specifically, all our scenarios include a global carbon price from 2020 at a value of $110 \$ / \mathrm{tCO}_{2}$ in US\$2010 kept constant throughout the century (Table 3). This provides a single indicator of mitigation effort which is comparable across scenarios and models. It should not be interpreted as implying that economy wide carbon pricing is the only instrument used to induce low carbon transformation particularly in the vehicle sector. We return to this point in the final discussion.

\subsection{Vehicle types}

Both IMAGE and MESSAGE resolve over ten different types of vehicle (Supplementary Information $\mathrm{H})$. To provide clear and interpretable insights, we show more aggregated results using four vehicle types: conventional vehicles (CV), battery electric vehicles (BEV), plug-in hybrid electric vehicles (PHEV) and other alternative fuel vehicles (other AFV). The relative attractiveness of each will be determined by endogenous model processes. However, as a generalisation based on their relative upfront costs, there tends to be a preference order from CVs to other AFVs (hybrids) to PHEV then BEVs, and then potentially to other AFVs (biofuels, hydrogen). However, the specific sequence with which different types of AFV substitute for CVs in the market will vary as a function of scenario and modelling assumptions. 


\section{Results for endogenous model formulation (in IMAGE)}

We introduce four improvements to the IMAGE model: (i) consumer heterogeneity; (ii) interdependent adopter groups; (iii) social influence effects; and (iv) cultural variation between regions. In presenting results we focus on evaluating the impact of these model improvements on our understanding of low-carbon vehicle transitions. In each case we summarise the main insights in bold, and then provide interpretive detail. In all results we show deployment rates expressed as \% shares of total passenger vehicle-kilometres.

\subsection{Impact of consumer heterogeneity}

\section{Consumer heterogeneity: Four adopter groups have distinctive initial risk premium values which} decline over the period to 2100 . Initial risk premiums and the speed of their decline towards zero show the extent to which each adopter group is attracted towards AFVs as novel vehicle technologies. Figure 5 shows the risk premium decline (left panel) and subsequent market share deployment (right panel) for BEVs in the USA as an example. For the EA group, the negative initial risk premium implies an attraction to novelty which declines as the market share of BEVs increases and their distinctiveness fades. In the EM, LM and LG groups, high positive initial risk premiums represent aversion to BEVs. The LG group is particularly averse with risk aversion adding well over $\$ 10,000$ of implicit cost to the upfront costs of a BEV. These risk premiums result in differentiated adoption rates of BEVs (and similarly for other types of AFV). BEV deployment begins by around 2075 (right panel) within the EA and EM groups only reflecting their reduced risk premiums towards zero. By $210037 \%$ of light duty vehicles are BEVs.
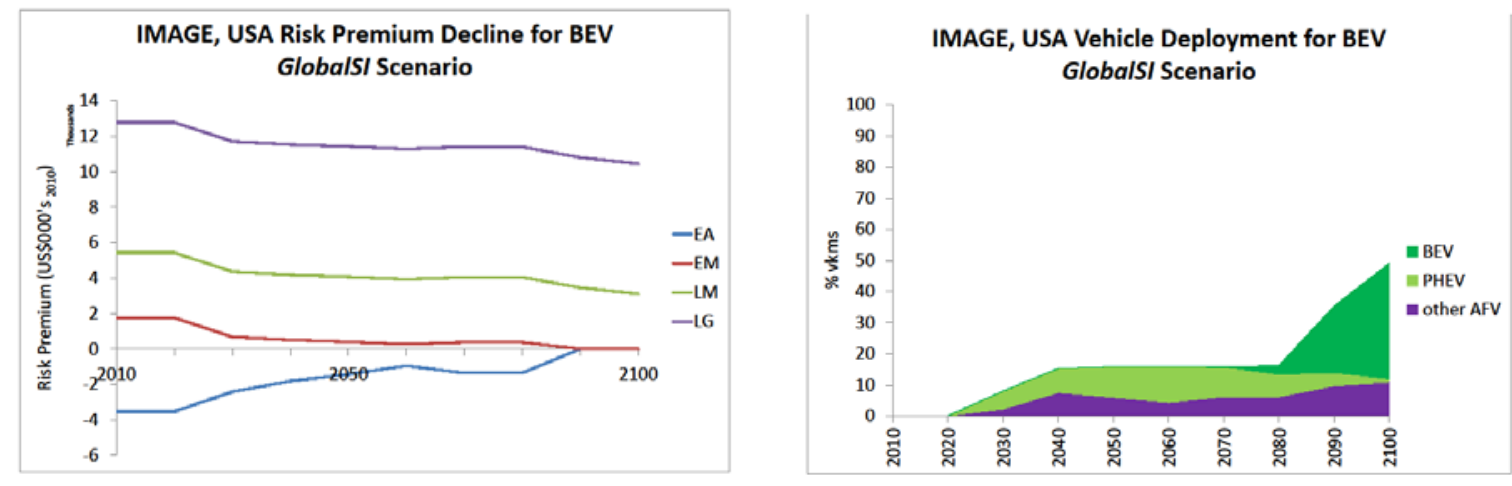

Figure 5 - Risk premium decline (left panel) and deployment (right panel) for BEVs in the USA in the IMAGE model, in the GlobalSI scenario

\subsection{Impact of social influence effects}

To determine the extent to which social influence accelerates the transition to AFVs, we compare the counterfactual NoSI scenario, which has static risk premiums and no social influence, with the GlobalSI scenario, which has declining risk premiums as a result of social influence between heterogeneous and interdependent adopter groups. We present global results as the GlobalSI scenario uses global average social influence effects. 

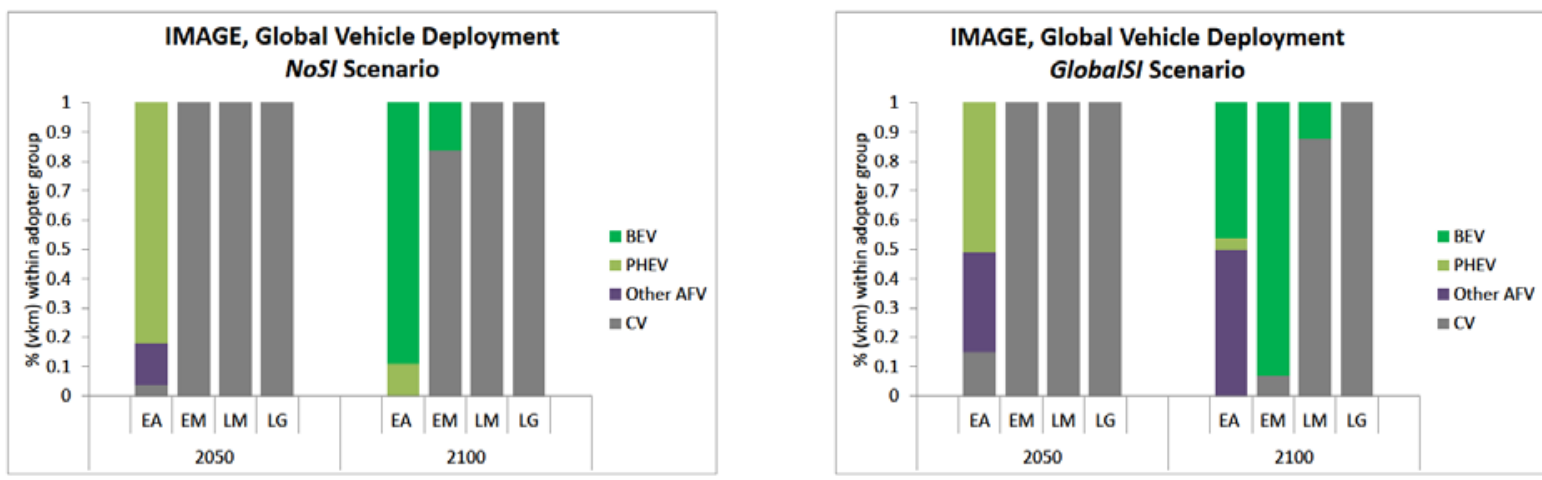

Figure 6 - The impact of social influence on vehicle deployment in 2050 and 2100 in the IMAGE model, comparing NoSI scenario (left panel) with GlobalSI scenario (right panel)

Interdependent adopter groups: Attractiveness of new vehicle types to early adopters leads to subsequent market growth and stronger differentiation with later adopting groups. The NOSI scenario shows the basic sequence of substitution from CVs to other AFVs to PHEV and BEV in the early adopter group (Figure 6 left panel). Initial risk premium values are held constant in the NoSI scenario so AFVs remain a higher cost alternative with widespread adoption only in this group by 2100. By comparison, social influence in the GlobalSI scenario between interdependent adopter groups results in more heterogeneous vehicle choices as early adopters seek to distinguish themselves, but in so doing reduce the perceived risks of novel vehicle types to later adopters. Differentiation linked to interdependent adoption is evident in 2100 as the EA group begins to adopt other AFVs (including hydrogen fuel cells) in response to the EM and LM groups' normalisation of BEVs as a mass market technology (Figure 6 right panel).

Social influence effects: The global diffusion of alternative fuel vehicles is accelerated by social influence as early adopters reduce perceived risks among mass market adopters. Social influence effects result in risk premiums declining more quickly in the LM group who respond to market share growth in earlier adopting EA and EM groups. Consequently BEVs deploy to a greater extent in later adopting groups (Figure 6 right panel). Table 4 shows a breakdown of vehicle deployment in 2050 and 2100 contrasting the NoSI and GlobalSI scenarios. Adding social influence effects stimulates deployment of AFVs overall. By $210052 \%$ of all passenger vehicle-kilometres $(\mathrm{vkm})$ are travelled in AFVs, compared to $22 \%$ in the NoSI scenario (Table 4).

Table 4 - Total vehicle deployment (\% vkm) in the IMAGE model, comparing NoSI scenario with GlobaISI scenario

\begin{tabular}{|c|c|c|c|c|}
\hline \multirow[b]{2}{*}{$\%$ vkm globally } & \multicolumn{2}{|c|}{ NoSI Scenario } & \multicolumn{2}{|c|}{ GlobalSI Scenario } \\
\hline & 2050 & 2100 & 2050 & 2100 \\
\hline CV & 0.85 & 0.78 & 0.86 & 0.48 \\
\hline AFV (all types) & 0.15 & 0.22 & 0.14 & 0.52 \\
\hline of which ... & & & & \\
\hline other AFV & 0.15 & 0.00 & 0.40 & 0.15 \\
\hline PHEV & 0.85 & 0.08 & 0.60 & 0.01 \\
\hline BEV & 0.00 & 0.92 & 0.00 & 0.83 \\
\hline
\end{tabular}

\subsection{Impact of cultural variation in social influence effects}

In the RegionaISI scenario we vary the size of the social influence effect to represent cultural differences between regions. To illustrate differences between the GlobalSI scenario and the RegionalSI scenario we contrast the USA as an example of a normative culture (strong social influence effect) with China as an example of a pragmatic culture (weak social influence effect).

Cultural variation: Regions with 'normative' cultures and receptiveness to social influence effects make a fuller transition to AFVs as risk aversion declines more rapidly following early adoption. Using BEVs as an example, Figure 7 (left panel) shows how a stronger social influence effect in the 
USA in the RegionalSI scenario results in risk premiums in all adopter groups moving towards zero more rapidly compared to the GlobalSI scenario. This reflects the stronger interdependencies between adopter groups, and the influence of early adopters. Figure 7 (right panel) shows the consequence on vehicle deployment in 2100. With more full deployment of BEVs across the EM and LM groups, BEVs lose their market distinctiveness as a result. This results in the EA group moving more strongly from PHEVs and BEVs onto other AFVs with greater novelty (including biofuels). An aggregate consequence is greater overall heterogeneity in vehicle choices between the adopter groups.
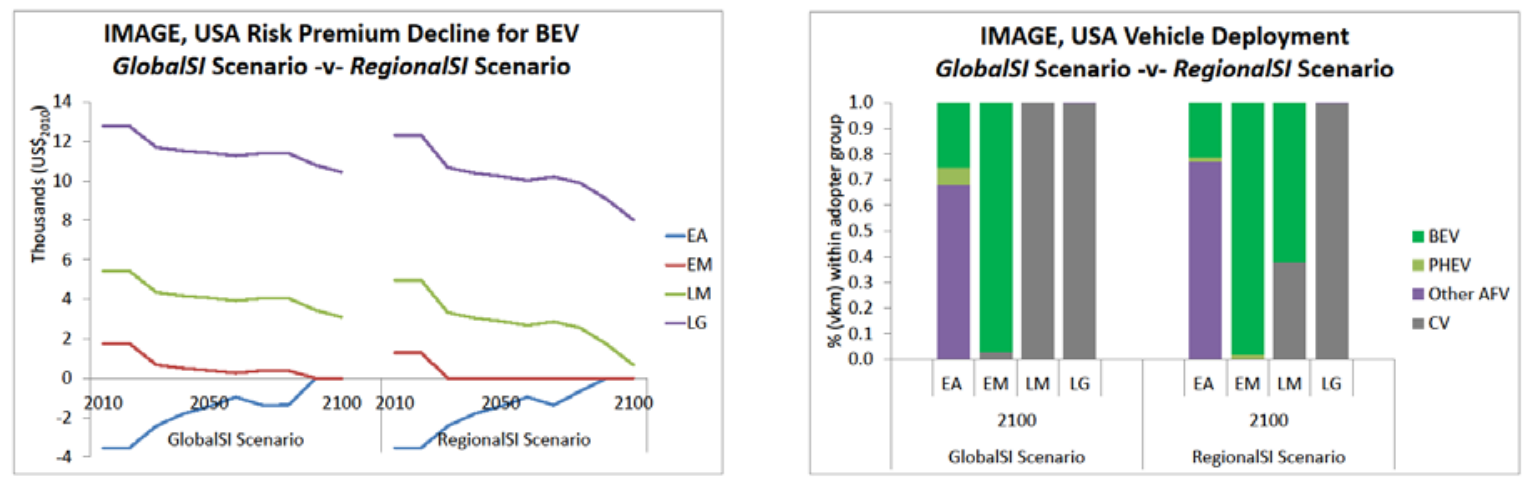

Figure 7 - Risk premium decline for BEVs in USA (left panel) and corresponding vehicle deployment by 2100 (right panel) in the IMAGE model, comparing GlobalSI scenario with RegionaISI scenario

\section{Cultural variation: Regions with 'pragmatic' cultures and resistance to social influence effects} make a slower transition to AFVs and show less differentiation between adopter groups. Using BEVs as an example, Figure 8 (left panel) shows the slower decline in risk premiums to zero across all adopter groups in China in the RegionalSI scenario as a result of weaker social influence effects. BEVs retain the attractiveness of novelty to the EA group right up until 2090; the EM, LM and LG groups all remain risk averse to BEVs through to 2100 . Figure 8 (right panel) shows some deployment of BEVs still occurs by 2100 in the EM group under the weaker social influences of the RegionalSI scenario. Across all adopter groups AFVs account for only about a third of all vehicle-kilometres by 2100 in the RegionalSI scenario, in contrast to over a half in the GlobalSI scenario.
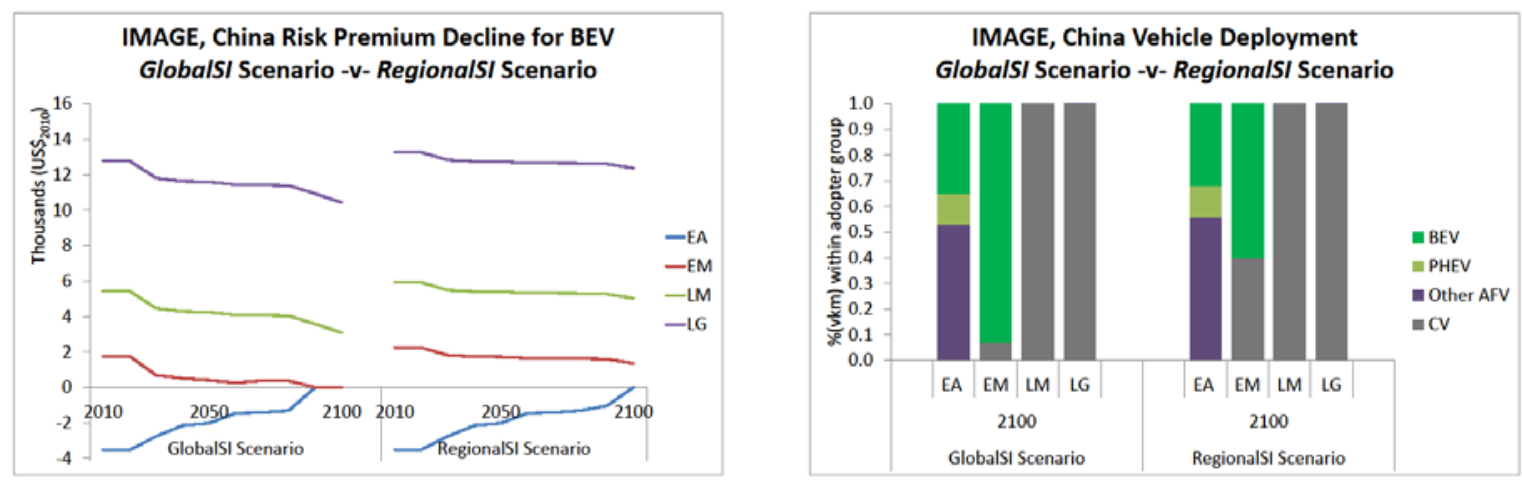

Figure 8 - Risk premium decline for BEVs in China (left panel) and corresponding vehicle deployment by 2100 (right panel) in the IMAGE model, comparing GlobalSI scenario with RegionaISI scenario

Cultural variation: Differentiated social influence effects change the rate and extent of AFV transition between regions but the aggregate global effect depends on other model processes. Not 
all differences between the USA and China in the RegionalSI scenario are attributable to regionalised social influence effects. It is important to reemphasise that other model assumptions and endogenous processes affect regional variation, including the extent to which changes in vehicle capital costs and fuel prices are assumed to be globally homogeneous. Given this caveat, in the GlobalSI scenario with a global average social influence effect, all AFVs have a $49 \%$ market share in the USA compared to $48 \%$ in China. In the RegionalSI scenario with social influence effects adjusted to reflect varying receptiveness to social influence within these very different cultures, all AFV' market share increase to $71 \%$ in the USA, but falls to $36 \%$ in China.

\section{Results for exogenous formulation (MESSAGE)}

We introduce three improvements to the MESSAGE model: (i) consumer heterogeneity; (ii) (ii) social influence effects; and (iii) cultural variation between regions. Unlike the endogenous formulation in the IMAGE model, the decline in risk premiums as a result of market experience is calculated externally, and fed back into the model. This model formulation effectively simulates social influence effects, but weakens the dynamic interdependence between adopter groups.

In presenting results we build on the insights on social influence and low-carbon vehicle transitions contributed by the IMAGE model analysis set out above. Rather than repeat key findings for the MESSAGE model, we identify any salient differences attributable either to the exogenous formulation of risk premium declines or to differences between MESSAGE and IMAGE.

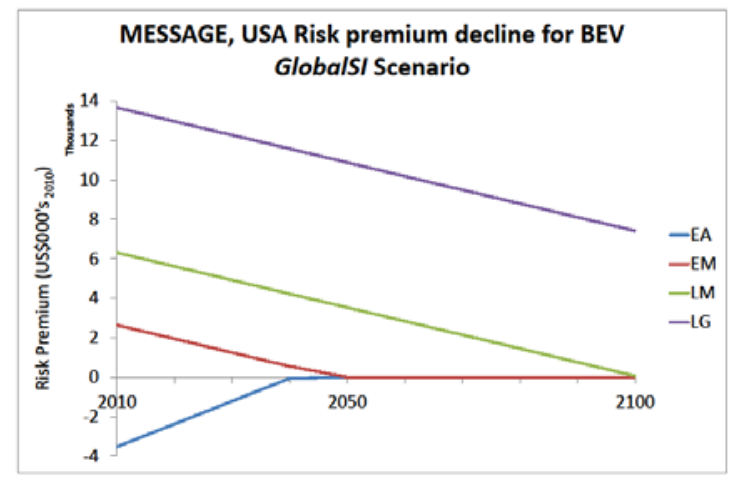

Figure 9 - Risk premium decline for BEVs in the USA in the MESSAGE model, in the GlobalSI scenario

Consumer heterogeneity: In the exogenous formulation of social influence, risk premiums for each adopter group decline linearly to zero. Figure 9 shows the linear decline to zero of risk premiums for $\mathrm{BEV}$ s in each adopter group as a function of BEV deployment generated by a base model run without social influence. The basic relationship between adoption propensity and market experience is otherwise very similar to the endogenous formulation.

Interdependent adopter groups: In the exogenous formulation of social influence, adopter groups do not respond directly to vehicle adoption in other groups. Although the exogenously specified risk premiums for each adopter group represents varying levels of risk aversion in the scenario storyline, risk premiums decline at similar rates determined by the global average social influence effect (GlobalSI scenario), this occurs independently of market share growth in other groups.

Despite this limitation as with the endogenous formulation we see similar patterns of AFV deployment across adopter groups. Taking BEVs as an example, Figure 10 (right panel) shows how BEVs are adopted in the EA and EM groups by 2050. As in the endogenous formulation, this is 
associated with increased deployment in the later adopting LM group by 2100 . By comparison, the NoSI scenario sees more adoption of BEVs in the EA group as risk premiums are negative and constant over the whole time period (Figure 10 left panel). Adding social influence effects to this counterfactual reduces BEV adoption as market deployment erodes the attractiveness of novelty to the EA group.
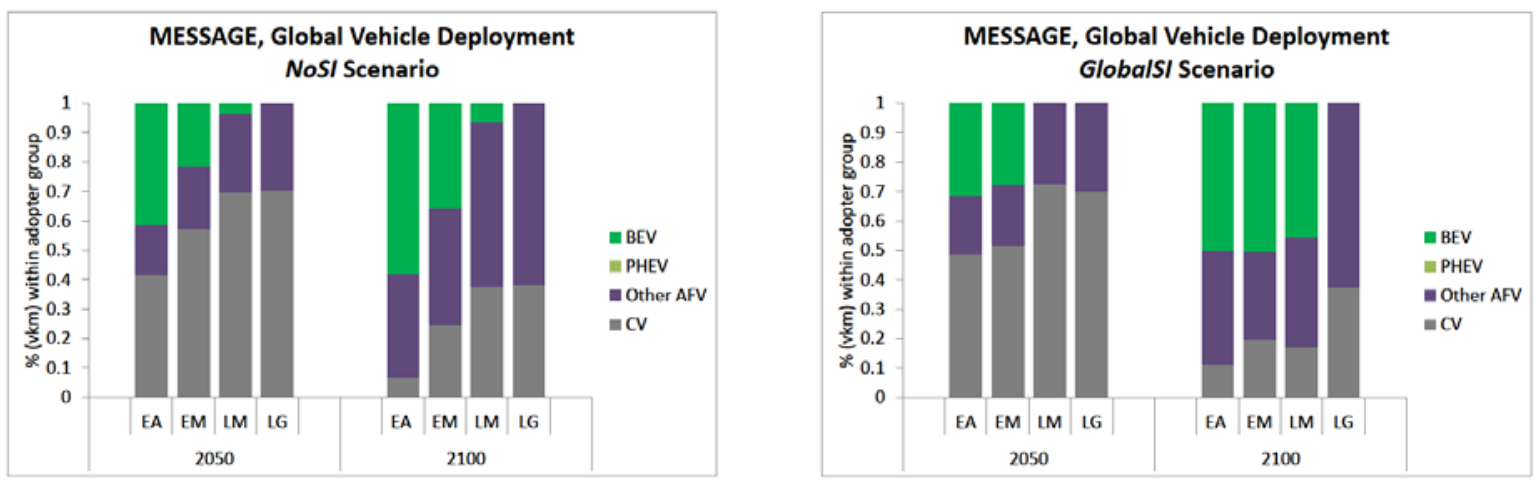

Figure 10 - The impact of social influence on vehicle deployment in 2050 and 2100 in the MESSAGE model, comparing NoSI scenario (left panel) with GlobalSI scenario (right panel)

Figure 10 shows results for the MESSAGE model equivalent to those shown in Figure 6 for the IMAGE model. There are some marked differences in the relative importance of different AFVs. By 2100 in the GlobalSI scenario, MESSAGE sees greater deployment of other AFVs (biofuel ICE, natural gas ICE and synfuel ICE hybrids) with BEVs dominating over PHEVs. Here BEVs are more attractive than PHEVs because they are much more efficient all the time, not just when operating in all-electric mode and they can be fully decarbonised which is important when there is a high carbon price. IMAGE on the other hand places much clearer emphasis on the transition to BEVs through PHEVs although with CVs still dominating in the LG adopter group by 2100 . This lack of emphasis on AFVs within IMAGE reflects the treatment of fossil based electric hybrids which IMAGE treats as more efficient CVs with zero risk premiums. Differences between model results are also partly due to the stronger interdependence of adopter groups in the endogenous formulation in IMAGE which leads to greater heterogeneity in vehicle choices. These differences also suggest that the vehicle choice in IMAGE is more sensitive to the addition of social influence effects than MESSAGE. In other words, the relative attractiveness of different vehicle types is more strongly determined in MESSAGE by capital and fuel cost differences.

The main difference between the models is that BEVs are adopted much earlier in MESSAGE compared to IMAGE. However isolating singular causes for differences in the outcomes of systems models is problematic (Kriegler et al., 2015) and our aim in this study is to understand how modelled systems respond to social influence effects which as we have shown are very similar between IMAGE and MESSAGE despite the marked differences in model solution architectures (recursive dynamic, optimisation) and in social influence model formulations (endogenous, exogenous).

\section{Discussion}

Our approach to improving the behavioural realism of global IAMs has a clear conceptual basis and strong empirical foundations. We introduce heterogeneous adopter segments with varying risk aversion to new technologies based on Rogers [2003] seminal synthesis of 50 years of diffusion research. We then parameterise the decline in risk aversion as experience with a new technology 
grows and is communicated socially using the results of a meta-analysis of over 20 studies of vehicle choice. We then incorporate these three elements of heterogeneous agents, risk aversion, and social influence effects into a model formulation which can be adapted for different types of global IAM including recursive dynamic models (e.g., IMAGE) and cost-optimisation models (e.g., MESSAGE). The endogenous formulation in IMAGE maintains the interdependency between adopter groups, and so is faithful to the conceptualisation in Rogers (2003). The exogenous formulation in MESSAGE is a work-around solution to the problem of endogenising adopter-interdependency in a perfect foresight model. Nevertheless, the basic modelled system response to social influence effects is still observed.

By modelling heterogeneous adopters, we find that early adopters play a critical role in seeding mass market adoption by reducing the perceived risks of a new vehicle type. By modelling social influence processes between interdependent adopters, we find that AFVs diffuse more rapidly but also more fully across different adopter types. By modelling cultural variation, we find significant differences in the deployment of AFVs between regions which are culturally divergent (such as USA and China). We therefore caution against overreliance on USA or OECD data as being representative for parameterisations in of all model regions.

In both models we see a slow transition to BEVs over the 100 year period which may be overtaken by real world changes already evident already in first-mover countries like Norway or Japan. Transitions in other parts of the world (e.g., sub-Saharan Africa) aggregated up to the global scale will necessarily be slower. However, it is important that model assumptions and calibration are continually updated in line with new evidence.

Our work develops and implements a transparent formulation for modelling social influence in widely-used global systems models based on strong conceptual and empirical foundations. However there are some important limitations which need to be taken into account when interpreting our findings.

Firstly the social influence effect we used was captured from a methodologically diverse range of empirical studies, and so may not represent a 'pure' social influence effect. The social influence effect size was derived from studies covering many different populations, 11 different countries and many different contexts.

Secondly our model formulation opens up the possibility for testing the effect of policy instruments designed specifically to harness or leverage social influence processes. However we are constrained in our ability to model social-influence based sectoral policies by the necessarily simplified framework of global systems models (hence our use of a global carbon price as a measure of climate policy). Although we find some small increase in AFV deployment in our sensitivity run of strong global social influence effects, this work could be developed to test policies that target early adopters to seed market diffusion by harnessing social influence effects, or policies that 'normalise' and reduce perceived risks with new vehicle technologies. Such policies can be both financial (e.g., fuel taxes, early adopter subsidies) as well as non-financial (e.g., efficiency standards, refueling infrastructure investments, car clubs, social marketing campaigns).

Thirdly our estimates of global risk premium values rely on studies in a small sample of countries. Due to these data limitations, we were only able to identify differences in risk premiums between USA and Europe, and as a global average. It seems likely that there is much greater variation between model regions in terms of risk aversion, particularly between developed and developing economies. However in earlier work we found varying risk premium starting values had little influence on overall deployment between regions. As a related point, we also use single risk premium estimates for all AFV technologies. Again this was due to data limitations in the diversity of 
empirical studies available, and again we would expect to see greater variation in potential adopters' risk aversion depending on vehicle type. This is an important area for further research, particularly with the rapid currently-observed growth in EVs.

Fourthly we model transitions between currently-available AFV technologies. This can result in the odd dynamic of early adopters switching back to 'old' technologies like the ICE later in the $21^{\text {st }}$ century as they become less desirable to the mass market. In reality, new vehicle types are highly likely to emerge over the next 80 years (e.g., autonomous vehicles) offering early adopters' genuine rather than nostalgic novelty.

Finally we implement our model formulations in global models with necessarily stylised representations of complex real-world transition processes (Trutnevyte, 2016). Although we improve the models' behavioural realism by incorporating heterogeneous adopter groups and social influence effects, the models still use cost-minimising decision functions, and in the case of MESSAGE, a system-wide social planner with perfect information who can find the least cost solution. Our results should not therefore be interpreted as being fully descriptively-realistic, but rather as cost-optimal solutions in a world populated by interdependent vehicle adopters with varying non-financial preferences. This is strongly supported both by theory (Rogers' diffusion of innovations) and by empirical evidence (the meta-analysis of social influence effects). In this respect, our work is consistent with Mundaca et al. (2010:332) who argues that improving the behavioural realism of global models is important, but only partially feasible, inherently selective, and needing empirical support.

\section{Conclusion}

The primary aim of this paper was to improve the behavioural realism of global IAMs, drawing on robust empirical evidence and a strong conceptual framework. This approach is entirely transparent in its parameterisations which are based on empirical studies. We demonstrate how to introduce social influences on consumer preferences for new vehicles. We developed and implemented a novel model formulation that is versatile for both myopic and perfect foresight IAMs. Our results show that both these formulations accurately represent the diffusion process according to Rogers (2003), although the endogenous formulation is a more direct implementation of theory.

We add intangible costs in the form of risk premiums with initial values that vary for each adopter group. These represent initial aversion (in the case of the EM, LM or LG groups) or attraction (in the case of the EA group) towards alternative fuel vehicles. These risk premiums decline as market share increases at a rate which is influenced by the social influence effect size. We see increased deployment of AFVs when we add social influence effects and when we increase the size of these to simulate sectoral policies targeting early adopters. We also adjust the social influence effect size to represent cultural variation in a country's receptiveness to social influence. We see levels of AFV deployment change accordingly with more deployment in normative cultures such as the USA, and less deployment in pragmatic cultures such as China. 
Bibliography

Achtnicht, M., Bühler, G., Hermeling, C. (2012) The impact of fuel availability on demand for alternative-fuel vehicles. Transportation Research Part D: Transport and Environment 17, 262-269. Adjemian, M.K., Cynthia Lin, C.Y., Williams, J. (2010) Estimating spatial interdependence in automobile type choice with survey data. Transportation Research Part A: Policy and Practice 44, 661-675.

Ahn, J., Jeong, G., Kim, Y. (2008) A forecast of household ownership and use of alternative fuel vehicles: A multiple discrete-continuous choice approach. Energy Economics 30, 2091-2104. Aini, M.S., Chan, S.C., Syuhaily, O. (2013) Predictors of technical adoption and behavioural change to transport energy-saving measures in response to climate change. Energy Policy 61, 1055-1062.

Arneth, A., Brown, C., Rounsevell, M.D.A. (2014) Global models of human decision-making for landbased mitigation and adaptation assessment. Nature Clim. Change 4, 550-557.

Avineri, E. (2012) On the use and potential of behavioural economics from the perspective of transport and climate change. Journal of Transport Geography 24, 512-521.

Axsen, J., Kurani, K.S. (2011) Interpersonal influence in the early plug-in hybrid market: Observing social interactions with an exploratory multi-method approach. Transportation Research Part D: Transport and Environment 16, 150-159.

Axsen, J., Mountain, D.C., Jaccard, M. (2009) Combining stated and revealed choice research to simulate the neighbor effect: The case of hybrid-electric vehicles. Resource and Energy Economics 31, 221-238.

Axsen, J., Orlebar, C., Skippon, S. (2013) Social influence and consumer preference formation for proenvironmental technology: The case of a U.K. workplace electric-vehicle study. Ecological Economics 95, 96-107.

Baltas, G., Saridakis, C. (2013) An empirical investigation of the impact of behavioural and psychographic consumer characteristics on car preferences: An integrated model of car type choice. Transportation Research Part A: Policy and Practice 54, 92-110.

Bass, F.M. (1969) A new product growth for model consumer durables. Management science 15, 215-227.

Batley, R.P., Toner, J.P., Knight, M.J. (2004) A mixed logit model of U.K. household demand for alternative-fuel vehicles. International Journal of Transport Economics 31, 55-77.

Bauernschuster, S., Falck, O. (2015) Culture, spatial diffusion of ideas and their long-lasting imprints-evidence from Froebel's kindergarten movement. Journal of Economic Geography 15, 601-630.

Borenstein, M.J., Hedges, L.V., Higgins, J.P., Rothstein, H.R. (2009) Introduction to meta-analysis. J Wiley \& Sons Ltd, Chichester.

Brand, C., Tran, M., Anable, J. (2012) The UK transport carbon model: An integrated life cycle approach to explore low carbon futures. Energy Policy 41, 107-124.

Brownstone, D., Bunch, D.S., Train, K. (2000) Joint mixed logit models of stated and revealed preferences for alternative-fuel vehicles. Transportation Research Part B: Methodological 34, 315338.

Bunch, D.S., Bradley, M., Golob, T.F., Kitamura, R., Occhiuzzo, G.P. (1993) Demand for clean-fuel vehicles in California: A discrete-choice stated preference pilot project. Transportation Research Part A: Policy and Practice 27, 237-253.

Calfee, J.E. (1985) Estimating the demand for electric automobiles using fully disaggregated probabilistic choice analysis. Transportation Research Part B: Methodological 19, 287-301. Caragliu, A., Nijkamp, P. (2016) Space and knowledge spillovers in European regions: the impact of different forms of proximity on spatial knowledge diffusion. Journal of Economic Geography 16, 749774. 
Cialdini, R.B., Kallgren, C.A., Reno, R.R., (1991) A focus theory of normative conduct: A theoretical refinement and reevaluation of the role of norms in human behavior, in: Mark, P.Z. (Ed.), Advances in Experimental Social Psychology. Academic Press, pp. 201-234.

Clarke, L., Jiang, K., Akimoto, K., Babiker, M., Blanford, G., Fisher-Vanden, K., Hourcade, J.-C., Krey, V., Kriegler, E., Löschel, A., McCollum, D., Paltsev, S., Rose, S., Shukla, P.R., Tavoni, M., Zwaan, B.v.d., Vuuren, D.P.v., (2014) Chapter 6: Assessing Transformation Pathways, Working Group III contribution to the IPCC 5th Assessment Report, Climate Change 2014: Mitigation of Climate Change. Cambridge University Press, Cambridge, UK and New York, NY, USA.

Cohen, J. (1992) A power primer. Psychological bulletin 112, 155-159.

Creutzig, F., Jochem, P., Edelenbosch, O.Y., Mattauch, L., Vuuren, D.P.v., McCollum, D., Minx, J. (2015) Transport: A roadblock to climate change mitigation? Science 350, 911-912.

Dagsvik, J.K., Wennemo, T., Wetterwald, D.G., Aaberge, R. (2002) Potential demand for alternative fuel vehicles. Transportation Research Part B: Methodological 36, 361-384.

de Mooij, M., Hofstede, G. (2002) Convergence and divergence in consumer behavior: Implications for international retailing. Journal of Retailing 78, 61-69.

Dimitropoulos, A., Rietveld, P., van Ommeren, J.N. (2013) Consumer valuation of changes in driving range: A meta-analysis. Transportation Research Part A: Policy and Practice 55, 27-45.

Dwyer, S., Mesak, H., Hsu, M. (2005) An Exploratory Examination of the Influence of National Culture on Cross-National Product Diffusion. Journal of International Marketing 13, 1-27.

Ekholm, T., Krey, V., Pachauri, S., Riahi, K. (2010) Determinants of household energy consumption in India. Energy Policy 38, 5696-5707.

Ewing, G., Sarigöllü, E. (2000) Assessing consumer preferences for clean-fuel vehicles: A discrete choice experiment. Journal of Public Policy and Marketing 19, 106-118.

Friedkin, N.E., Johnsen, E.C. (1990) Social influence and opinions. The Journal of Mathematical Sociology 15, 193-206.

Gaker, D., Zheng, Y., Walker, J. (2010) Experimental economics in transportation: A focus on social influences and the provision of information. Transportation Research Record.

Girod, B., van Vuuren, D., Grahn, M., Kitous, A., Kim, S., Kyle, P. (2013) Climate impact of transportation A model comparison. Climatic Change 118, 595-608.

Goetzke, F., Weinberger, R. (2012) Separating contextual from endogenous effects in automobile ownership models. Environment and Planning A 44, 1032-1046.

Griffith, D.A., Yalcinkaya, G., Rubera, G. (2014) Country-Level Performance of New Experience Products in a Global Rollout: The Moderating Effects of Economic Wealth and National Culture. Journal of International Marketing 22, 1-20.

Grinblatt, M., Keloharju, M., Ikäheimo, S. (2008) Social influence and consumption: Evidence from the automobile purchases of neighbors. Review of Economics and Statistics 90, 735-753.

Griskevicius, V., Tybur, J., Van den Bergh, B. (2010) Going Green to Be Seen: Status, Reputation, and Conspicuous Conservation. Journal of Personality and Social Psychology.

Hackbarth, A., Madlener, R. (2013) Consumer preferences for alternative fuel vehicles: A discrete choice analysis. Transportation Research Part D: Transport and Environment 25, 5-17.

Heutel, G., Muehlegger, E., (2010) Consumer learning and hybrid vehicle adoption, Working Paper Series, Harvard Kennedy School.

Hidrue, M.K., Parsons, G.R., Kempton, W., Gardner, M.P. (2011) Willingness to pay for electric vehicles and their attributes. Resource and Energy Economics 33, 686-705.

Hofstede, G.H. (2001) Culture's consequences : comparing values, behaviors, institutions, and organizations across nations. Thousand Oaks, Calif. ; London : SAGE, 2001

2nd ed.

Horne, M., Jaccard, M., Tiedemann, K. (2005) Improving behavioral realism in hybrid energyeconomy models using discrete choice studies of personal transportation decisions. Energy Economics 27, 59-77. 
Hsu, C.-I., Li, H.-C., Lu, S.-M. (2013) A dynamic marketing model for hybrid electric vehicles: A case study of Taiwan. Transportation Research Part D: Transport and Environment 20, 21-29.

Hutter, K., Hautz, J., Dennhardt, S., Füller, J. (2013) The impact of user interactions in social media on brand awareness and purchase intention: the case of MINI on Facebook. Journal of Product \& Brand Management 22, 342-351.

Jansson, J., Marell, A., Nordlund, A. (2010) Green consumer behavior: determinants of curtailment and eco - innovation adoption. Journal of Consumer Marketing 27, 358-370.

Keppo, I., Strubegger, M. (2010) Short term decisions for long term problems - The effect of foresight on model based energy systems analysis. Energy 35, 2033-2042.

Krey, V. (2014) Global energy-climate scenarios and models: a review. Wiley Interdisciplinary Reviews: Energy and Environment 3, 363-383.

Kriegler, E., Petermann, N., Krey, V., Schwanitz, V.J., Luderer, G., Ashina, S., Bosetti, V., Eom, J., Kitous, A., Méjean, A., Paroussos, L., Sano, F., Turton, H., Wilson, C., Van Vuuren, D.P. (2015) Diagnostic indicators for integrated assessment models of climate policy. Technological Forecasting and Social Change 90, 45-61.

Kulkarni, G., Ratchford, B.T., Kannan, P.K. (2012) The impact of online and offline information sources on automobile choice behavior. Journal of Interactive Marketing 26, 167-175.

Lavine, H., Latané, B. (1996) A Cognitive-Social Theory of Public Opinion Dynamic Social Impact and Cognitive Structure. Journal of Communication 46, 48-56.

Lebeau, K., Van Mierlo, J., Lebeau, P., Mairesse, O., Macharis, C. (2012) The market potential for plug-in hybrid and battery electric vehicles in Flanders: A choice-based conjoint analysis.

Transportation Research Part D: Transport and Environment 17, 592-597.

Lin, Z., Dong, J., Greene, D.L. (2013) Hydrogen vehicles: Impacts of DOE technical targets on market acceptance and societal benefits. International Journal of Hydrogen Energy 38, 7973-7985.

Lin, Z., Greene, D. (2009) A plug in hybrid consumer choice model with detialed market segmentation. Oak Ridge National Laboratory.

Lipsey, R.G., Carlaw, K.I., Bekar, C.T. (2005) Economic Transformations: General Purpose Technologies and Long-term Economic Growth. Oxford University Press, Oxford, UK.

Manski, C.F. (1993) Identification of endogenous social effects: The reflection problem. The Review of Economic Studies 60, 531-542.

Mattauch, L., Ridgway, M., Creutzig, F. (2015) Happy or liberal? Making sense of behavior in transport policy design. Transportation Research Part D: Transport and Environment.

Mau, P., Eyzaguirre, J., Jaccard, M., Collins-Dodd, C., Tiedemann, K. (2008) The 'neighbor effect':

Simulating dynamics in consumer preferences for new vehicle technologies. Ecological Economics 68, 504-516.

McCollum, D., Wilson, C., Pettifor, H., Ramea, K., Krey, V., Riahi, K., Bertram, C., Lin, Z., Edelenbosch, O., Fujisawa, S. (2016) Improving the behavioural realism of global integrated assessment models:

An application to consumers' vehicle choices. Transportation Research Part D: Transport and

Environment In Proof. Accepted for Submission. Available

online http://doi.org/10.1016/j.trd.2016.04.003.

McShane, B.B., Bradlow, E.T., Berger, J. (2012) Visual influence and social groups. Journal of Marketing Research (JMR) 49, 854-871.

Meade, N., Islam, T. (2006) Modelling and forecasting the diffusion of innovation - A 25-year review. International Journal of Forecasting 22, 519-545.

Mercure, J.-F., Pollitt, H., Bassi, A.M., Viñuales, J.E., Edwards, N.R. (2016) Modelling complex systems of heterogeneous agents to better design sustainability transitions policy. Global Environmental Change 37, 102-115.

Mercure, J.F., Lam, A. (2015) The effectiveness of policy on consumer choices for private road passenger transport emissions reductions in six major economies. Environmental Research Letters 10, 064008. 
Minkov, M., Hofstede, G. (2012) Hofstede's fifth dimension: New evidence from the World Values Survey. Journal of Cross-Cultural Psychology 43, 3-14.

Mohammad Reza, J., Neda, S. (2012) The effect of electronic word of mouth on brand image and purchase intention. Marketing Intelligence \& Planning 30, 460-476.

Moons, I., De Pelsmacker, P. (2012) Emotions as determinants of electric car usage intention. Journal of Marketing Management 28, 195-237.

Mundaca, L., Neij, L., Worrell, E., McNeil, M. (2010) Evaluating energy efficiency policies with energyeconomy models. Annual Review of Environment and Resources 35, 305-344.

Nilsson, M., Nykvist, B. (2016) Governing the electric vehicle transition - Near term interventions to support a green energy economy. Applied Energy.

Peres, R., Muller, E., Mahajan, V. (2010) Innovation diffusion and new product growth models: A critical review and research directions. International Journal of Research in Marketing 27, 91-106.

Peterson, R.A., Brown, S.P. (2005) On the use of beta coefficients in meta-analysis. Journal of Applied Psychology 90, 175-181.

Pettifor, H., Wilson, C., Axsen, J., Abrahamse, W., Anable, J. (2017) Social influence in the global diffusion of alternative fuel vehicles - A meta-analysis. Journal of Transport Geography 62, 247-261. Riahi, K., Dentener, F., Gielen, D., Grubler, A., Jewell, J., Klimont, Z., Krey, V., McCollum, D., Pachauri, S., Rao, S., van Ruijven, B., van Vuuren, D.P., Wilson, C., (2012) Energy Pathways for Sustainable Development, The Global Energy Assessment. Cambridge University Press, Cambridge, UK.

Rivers, N., Jaccard, M. (2005) Combining Top-Down and Bottom-Up Approaches To Energy-Economy Modeling Using Discrete Choice Methods. Energy Journal 26, 83-106.

Rogers, E.M. (2003) Diffusion of Innovations. Free Press, New York.

Rosenthal, R., DiMatteo, M.R. (2001) Meta-analysis: Recent developments in quantitative methods for literature reviews. Annual Review of Psychology 52, 59-82.

Schuitema, G., Anable, J., Skippon, S., Kinnear, N. (2013) The role of instrumental, hedonic and symbolic attributes in the intention to adopt electric vehicles. Transportation Research Part A: Policy and Practice 48, 39-49.

Sha, Y., Mantian, H., Winer, R.S., Assael, H., Xiaohong, C. (2012) An empirical study of word-of-mouth generation and consumption. Marketing Science 31, 952-963.

Shemesh, J., Zapatero, F., (2014) Thou shalt not covet thy (suburban) neighbour's car, 2011 annual meeting of the academy of behavioural finance \& economics.

Sims, R., Schaeffer, R., Creutzig, F., Cruz-Núñez, X., D’Agosto, M., Dimitriu, D., Figueroa Meza, M.J., Fulton, L., Kobayashi, S., Lah, O., McKinnon, A., Newman, P., Ouyang, M., Schauer, J.J., Sperling, D., Tiwari, G., (2014) Transport

in: Edenhofer, O., R. Pichs-Madruga, Y. Sokona, E. Farahani, S. Kadner, K. Seyboth, A. Adler,, I. Baum, S.B., P. Eickemeier, B. Kriemann, J. Savolainen, S. Schlömer, C. von Stechow, T. Zwickel and J.C. Minx (eds.). (Eds.), Climate Change Contribution of Working Group III to the Fifth Assessment Report of the Intergovernmental Panel on Climate Change. Cambridge University Press, Cambridge.

Stehfest, E., van Vuuren, D., Kram, T., Bouwman, L., Alkemade, R., Bakkenes, M., Biemans, H., Bouwman, A., den Elzen, M., Janse, J., Lucas, P., van Minnen, J., Müller, M., Prins, A. (2014) Integrated Assessment of Global Environmental Change with IMAGE 3.0. Model description and policy applications. Netherlands Environmental Assessment Agency (PBL), The Hague.

Trutnevyte, E. (2016) Does cost optimization approximate the real-world energy transition? Energy 106, 182-193.

van Everdingen, Y., Fok, D., Stremersch, S. (2009) Modeling Global Spillover of New Product Takeoff. Journal of Marketing Research (JMR) 46, 637-652.

Wiedmann, K.-P., Hennigs, N., Pankalla, L., Kassubek, M., Seegebarth, B. (2011) Adoption barriers and resistance to sustainable solutions in the automotive sector. Journal of Business Research 64, 1201-1206.

Zhang, Y., Yu, Y., Zou, B. (2011) Analyzing public awareness and acceptance of alternative fuel vehicles in China: The case of EV. Energy Policy 39, 7015-7024. 
Zhu, X., Chao, L. (2013) Investigating the neighborhood effect on hybrid vehicle adoption.

Transportation Research Record: Journal of the Transportation Research Board, 37-44.

Ziegler, A. (2012) Individual characteristics and stated preferences for alternative energy sources and propulsion technologies in vehicles: A discrete choice analysis for Germany. Transportation Research Part A: Policy and Practice 46, 1372-1385. 
Modelling Social Influence and Cultural Variation in Global Low-Carbon Vehicle Transitions

\section{Supplementary Information}

\begin{tabular}{|l|l|}
\hline Ref & Description \\
\hline A & Meta-analysis and calculation of social influence effect size \\
\hline B & Calculation of initial risk premium values \\
\hline C & Calculation of regional variation in social influence effect sizes \\
\hline D & Calculation of decline function for endogenous model formulation and input equations \\
\hline E & Calculation of decline function for exogenous model formulation \\
\hline F & Technology grouping used in models \\
\hline G & Results of sensitivity run StrongSI scenario \\
\hline
\end{tabular}




\section{Supplementary Information A - Meta analysis and calculation of social influence effect size}

The decline function for social influence effect size $(+1 \bar{\sigma} M K=0.241 * \bar{\sigma} R P)$ is derived from this meta-analysis of studies examining effect of social influences on vehicle choice.

To derive an effect size for the effect of social influence on vehicle choice we conducted a systematic literature review using a comprehensive set of defined keywords to identify studies that empirically examined this relationship. We identified a total of 43 studies from 16 different countries spanning the period 1967 to 2014 . We reviewed the conceptual framework, methodology and results of each study. We identified three types of social influence that were distinguished empirically: (1) interpersonal communication (2) neighbourhood effect (3) social norms. Interpersonal communication emphasises the importance of social networks through which information about a new vehicle is exchanged, and potential adopters search for advice and learned experience from trusted others (Axsen et al., 2013; Baltas and Saridakis, 2013; Gaker et al., 2010). Neighbourhood effect emphasises the importance of information gained by observing new vehicles being used by others in close proximity (Adjemian et al., 2010; Grinblatt et al., 2008). Social norms emphasise the importance of the perceived commonness of a new vehicle, as well as any approval (or disapproval) associated with its use: people are concerned with what others think (McShane et al., 2012; Peres et al., 2010). Of the 43 studies identified through systematic literature review as measuring social influence effects on vehicle choice (conventional vehicles as well as AFVs), 29 studies were initially considered suitable for meta-analysis as they reported quantitative measures of social influence. However, six studies were excluded because they did not report specific effect sizes for social influence. For example, various studies using discrete choice experiments and agent-based model simulations report post-estimation statistics such as willingness to pay (WTP) but these are not convertible to a comparable metric. A further two studies were excluded because they report outcome statistics that are not comparable to other studies (e.g., paired t-tests, rankings). A total of 21 studies were included in the meta-analysis and are summarised in Table 5. Studies were included regardless of whether social influence was found to be significant; non-significant results were found in two studies.

Table 5 - Empirical studies included in the meta-analysis of social influence on vehicle choice $(\mathrm{N}=21)$

\begin{tabular}{|l|l|l|l|l|l|}
\hline Author(s) & Country & $\begin{array}{l}\text { Social influence } \\
\text { type }\end{array}$ & Target group & $\mathrm{N}$ & Outcome measure \\
\hline $\begin{array}{l}\text { Axsen et al. } \\
\text { (2013) }\end{array}$ & UK & $\begin{array}{l}\text { Interpersonal } \\
\text { communication }\end{array}$ & Workplace staff & 170 & $\begin{array}{l}\text { Vehicle choice between AFV and } \\
\text { conventional vehicle given } \\
\text { interactions with co-workers }\end{array}$ \\
\hline $\begin{array}{l}\text { Baltas and } \\
\text { Saridakis } \\
\text { (2013) }\end{array}$ & Greece & $\begin{array}{l}\text { Interpersonal } \\
\text { communication }\end{array}$ & Car drivers & 1,357 & $\begin{array}{l}\text { Choice between 12 alternative } \\
\text { fuel cars given pre-purchase } \\
\text { information from friends and } \\
\text { family. }\end{array}$ \\
\hline $\begin{array}{l}\text { Hsu et al. } \\
\text { (2013) }\end{array}$ & Taiwan & $\begin{array}{l}\text { Interpersonal } \\
\text { communication }\end{array}$ & $\begin{array}{l}\text { Car owners and } \\
\text { non-car owners }\end{array}$ & 1,594 & $\begin{array}{l}\text { Choice between vehicles given } \\
\text { influences of social networks } \\
\text { spreading eWOM }\end{array}$ \\
\hline $\begin{array}{l}\text { Hutter et al. } \\
\text { (2013) }\end{array}$ & Germany & $\begin{array}{l}\text { Interpersonal } \\
\text { communication }\end{array}$ & $\begin{array}{l}\text { Potential } \\
\text { customers of } \\
\text { specific } \\
\text { make/model }\end{array}$ & 311 & $\begin{array}{l}\text { Purchase intention as a function } \\
\text { of word of mouth }\end{array}$ \\
\hline $\begin{array}{l}\text { Kulkarni et al. } \\
\text { (2012) }\end{array}$ & USA & $\begin{array}{l}\text { Interpersonal } \\
\text { communication }\end{array}$ & $\begin{array}{l}\text { Buyers of family } \\
\text { sedans }\end{array}$ & 886 & $\begin{array}{l}\text { Choice between 24 family cars } \\
\text { given recommendations from } \\
\text { other consumers through online } \\
\text { resources }\end{array}$ \\
\hline $\begin{array}{l}\text { Mohammad } \\
\text { Reza and } \\
\text { Neda (2012) }\end{array}$ & Iran & $\begin{array}{l}\text { Interpersonal } \\
\text { communication }\end{array}$ & $\begin{array}{l}\text { Potential } \\
\text { customers of } \\
\text { specific }\end{array}$ & 341 & $\begin{array}{l}\text { Purchase intention as a function } \\
\text { of word of mouth }\end{array}$ \\
\hline
\end{tabular}




\begin{tabular}{|c|c|c|c|c|c|}
\hline & & & make/model & & \\
\hline $\begin{array}{l}\text { Sha et al. } \\
(2012)\end{array}$ & UK & $\begin{array}{l}\text { Interpersonal } \\
\text { communication }\end{array}$ & $\begin{array}{l}\text { Purchasers of } \\
\text { new vehicle }\end{array}$ & 4,544 & $\begin{array}{l}\text { Car ownership and propensity to } \\
\text { use word of mouth }\end{array}$ \\
\hline $\begin{array}{l}\text { Zhang et al. } \\
\text { (2011) }\end{array}$ & China & $\begin{array}{l}\text { Interpersonal } \\
\text { communication }\end{array}$ & $\begin{array}{l}\text { Driving school } \\
\text { pupils }\end{array}$ & 299 & $\begin{array}{l}\text { Willingness to adopt AFV as } \\
\text { function of peer effects }\end{array}$ \\
\hline $\begin{array}{l}\text { Adjemian et } \\
\text { al. (2010) }\end{array}$ & USA & $\begin{array}{l}\text { Neighbourhood } \\
\text { effect }\end{array}$ & $\begin{array}{l}\text { Households in } \\
\text { given census } \\
\text { region }\end{array}$ & 560 & $\begin{array}{l}\text { Purchase of specific car body } \\
\text { type given behaviour of others in } \\
\text { the same neighbourhood }\end{array}$ \\
\hline $\begin{array}{l}\text { Goetzke and } \\
\text { Weinberger } \\
(2012) \\
\end{array}$ & USA & $\begin{array}{l}\text { Neighbourhood } \\
\text { effect }\end{array}$ & $\begin{array}{l}\text { People who } \\
\text { would buy small } \\
\text { vehicle }\end{array}$ & 3,322 & $\begin{array}{l}\text { Car ownership as a function of } \\
\text { characteristics of the } \\
\text { neighbourhood }\end{array}$ \\
\hline $\begin{array}{l}\text { Grinblatt et } \\
\text { al. (2008) }\end{array}$ & Finland & $\begin{array}{l}\text { Neighbourhood } \\
\text { effect }\end{array}$ & $\begin{array}{l}\text { Purchasers of } \\
\text { new vehicle }\end{array}$ & 211,173 & $\begin{array}{l}\text { Car purchase as a function of } \\
\text { purchases of near neighbours }\end{array}$ \\
\hline $\begin{array}{l}\text { Heutel and } \\
\text { Muehlegger } \\
\text { (2010) }\end{array}$ & USA & $\begin{array}{l}\text { Neighbourhood } \\
\text { effect }\end{array}$ & $\begin{array}{l}\text { Purchasers of } \\
\text { new vehicle }\end{array}$ & 4,630 & $\begin{array}{l}\text { AFV sales in given state as a } \\
\text { function of increased visibility } \\
\text { effects }\end{array}$ \\
\hline $\begin{array}{l}\text { McShane et } \\
\text { al. (2012) }\end{array}$ & USA & $\begin{array}{l}\text { Neighbourhood } \\
\text { effect }\end{array}$ & $\begin{array}{l}\text { Purchasers of } \\
\text { new vehicle }\end{array}$ & 1,000 & $\begin{array}{l}\text { Car purchase behaviour based on } \\
\text { within eyeshot purchases of near } \\
\text { neighbours }\end{array}$ \\
\hline $\begin{array}{l}\text { Shemesh and } \\
\text { Zapatero } \\
(2014)\end{array}$ & USA & $\begin{array}{l}\text { Neighbourhood } \\
\text { effect }\end{array}$ & $\begin{array}{l}\text { People who } \\
\text { purchased } \\
\text { new/used car }\end{array}$ & 8,981 & $\begin{array}{l}\text { Intentions towards purchase } \\
\text { luxury car given purchase } \\
\text { behaviour of near neighbours }\end{array}$ \\
\hline $\begin{array}{l}\text { Zhu and Chao } \\
\text { (2013) }\end{array}$ & USA & $\begin{array}{l}\text { Neighbourhood } \\
\text { effect }\end{array}$ & $\begin{array}{l}\text { Vehicle owners } \\
\text { including AFV }\end{array}$ & 15,884 & $\begin{array}{l}\text { Purchase choice of AFV as a } \\
\text { function of behaviour of near } \\
\text { neighbours }\end{array}$ \\
\hline $\begin{array}{l}\text { Aini et al. } \\
(2013)\end{array}$ & Malaysia & Social norms & $\begin{array}{l}\text { Government } \\
\text { workers }\end{array}$ & 201 & $\begin{array}{l}\text { Purchase intention towards AFVs } \\
\text { moderated by individual } \\
\text { subjective norms }\end{array}$ \\
\hline $\begin{array}{l}\text { Gaker et al. } \\
(2010)\end{array}$ & USA & Social norms & Students & 312 & $\begin{array}{l}\text { Choice between AFV and } \\
\text { conventional vehicle given } \\
\text { knowledge of behaviour of others }\end{array}$ \\
\hline $\begin{array}{l}\text { Jansson et al. } \\
(2010)\end{array}$ & Sweden & Social norms & Car owners & 1,832 & $\begin{array}{l}\text { Willingness to adopt a AFV as a } \\
\text { function of subjective norms }\end{array}$ \\
\hline $\begin{array}{l}\text { Moons and } \\
\text { De } \\
\text { Pelsmacker } \\
(2012)\end{array}$ & Belgium & Social norms & Students & 1,202 & $\begin{array}{l}\text { Intention to adopt AFV as a } \\
\text { function of subjective norms }\end{array}$ \\
\hline $\begin{array}{l}\text { Schuitema et } \\
\text { al. (2013) }\end{array}$ & UK & Social norms & Car owners & 2,729 & $\begin{array}{l}\text { Intention to adopt AFV as } \\
\text { function of symbolic benefits }\end{array}$ \\
\hline $\begin{array}{l}\text { Wiedmann et } \\
\text { al. (2011) }\end{array}$ & Germany & Social norms & Car owners & 480 & $\begin{array}{l}\text { Social risk involved in ownership } \\
\text { of AFV }\end{array}$ \\
\hline
\end{tabular}

Meta-analysis involves the identification of a comparable metric for each study, and the weighting of relative effect size according to sample size. The comparable metric we identify between studies is the correlation coefficient ( $r$ ) since all studies report either betas, log odds (probits), or chi-square statistics. These are easily converted into a common metric: Fisher's $\mathrm{Zr}$ (Borenstein et al., 2009). For studies reporting only standardised beta coefficients $(\beta)$, these are used as a substitute for correlation coefficients $(r)$. We assume that all regression coefficients reported are standardised, even if this is not made clear in the study. Use of standardised regression coefficients as a metric of effect size is common within the social sciences (Borenstein et al., 2009; Rosenthal and DiMatteo, 2001). Peterson and Brown (2005) show ' $\beta$ ' and ' $r$ ' are highly correlated. Effect sizes for each study are therefore calculated using either betas and sample size, or odds ratios and standard errors, depending on which are reported (Peterson and Brown, 2005).

Figure 11 displays a forest plot with effect sizes for each study, the overall average effect size (0.241) and accompanying statistics from a random effects meta-analysis model. All individual effect sizes are positive and all but two are significant at $|p| \leq 0.05$. 


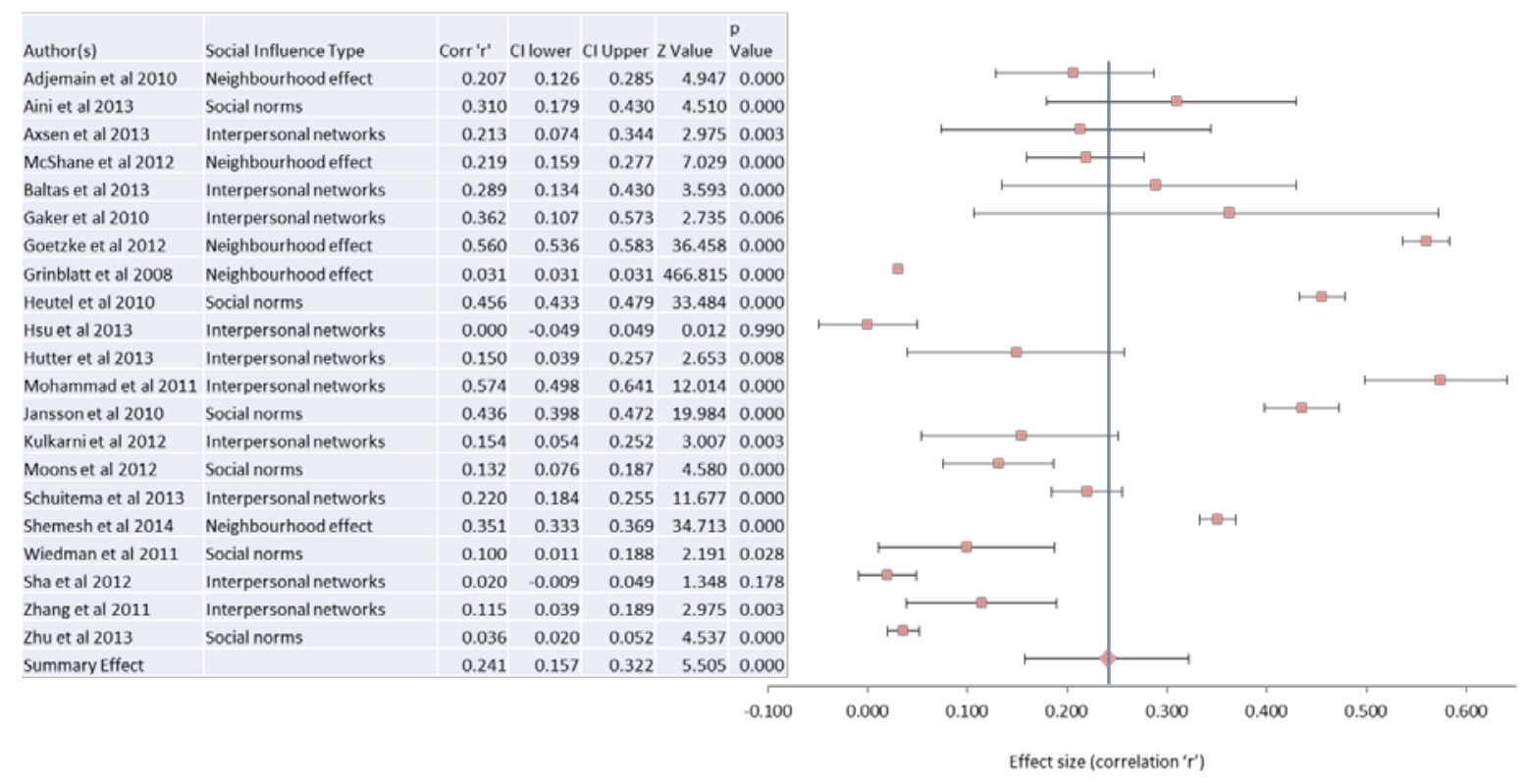

Figure 11 - Forest Plot of Social Influence Effect Sizes

Our meta-analysis of the 21 studies revealed an average effect size measured by the correlation coefficient ' $r$ ' of $0.241(95 \% \mathrm{Cl}[0.157,0.322], Z=5.505,|p| \leq 0.000)$. This is shown by the vertical line in Figure 11 and is considered to be a small to medium effect in meta-analysis (Cohen, 1992).

The measurement of social influences is subject to the endogeneity problem discussed by Manski (1993) whereby social influence effects are difficult to identify, particularly when modelling the behaviour of similar individuals (such as in the case of neighbourhood effects). This endogeneity exists because we cannot be certain whether the effects observed occur because $A$ influences $B$, or because $A$ and $B$ are similar types. The social influence effect size derived from the meta-analysis was based on many different study designs. Some effect sizes were based on surveys which directly recorded individuals own opinions as to whom influences their purchase decisions, modelled then as part of a regression, controlling for demographic similarities between respondents e.g., . Axsen and Kurani (2011). Those studies which compared the vehicle choices of people living in similar post code areas (neighbourhood effect studies) also controlled for this endogeneity using for example spatial lags or instrumental variables e.g., . Goetzke and Weinberger (2012) and Grinblatt et al. (2008). 


\section{Supplementary Information B - Calculation of initial risk premium values}

To calculate the initial risk premium values for adopter groups we draw on the empirical literature. Conducting a synthesis of comparative discrete choice studies measuring stated preferences for AFVs ( $n=14$ studies) we calculate willingness to pay (WTP) ratios in accordance with Batley et al. (2004) as the ratio between consumer utility/disutility for an AFV and price (adjusted to US\$2010). Consumer utility is captured in discrete choice experiments by the alternative specific constant (APC) which measures base utility for an AFV (whilst controlling for all other attributes such as fuel costs, vehicle range and refuelling infrastructure).

We take WTP ratios from empirical studies which themselves are derived from a distribution and we use these as independent point estimates assuming they are representative of an overall population. We use this distribution to estimate mean and standard deviation from which we estimate specific values for each adopter group. The main assumptions made here are (a) the distribution is normally distributed and this is confirmed by a Shapiro Wilk W test for normality and (b) the propensity for consumers to adopt is consistent with Roger's diffusion curve.

Table 6 - Discrete choice studies used to calculate WTP AFV

\begin{tabular}{|l|c|c|l|}
\hline Author/year & $\begin{array}{l}\text { Fuel Technologies included in } \\
\text { study (n) }\end{array}$ & $\begin{array}{l}\text { WTP estimations } \\
(\mathrm{n})\end{array}$ & $\begin{array}{l}\text { Country from which sample } \\
\text { derived }\end{array}$ \\
\hline Achtnicht et al. (2012) & 5 & 5 & Germany \\
\hline Ahn et al. (2008) & 3 & 3 & South Korea \\
\hline Axsen et al. (2009) & 1 & 1 & Canada \\
\hline Brownstone et al. (2000) & 3 & 3 & USA \\
\hline Bunch et al. (1993) & 3 & 3 & USA \\
\hline Calfee (1985) & 1 & 1 & USA \\
\hline Dagsvik et al. (2002) & 2 & 2 & Norway \\
\hline Ewing and Sarigöllü (2000) & 2 & 2 & Canada \\
\hline Gaker et al. (2010) & 1 & 1 & USA \\
\hline $\begin{array}{l}\text { Hackbarth and Madlener } \\
\text { (2013) }\end{array}$ & 5 & 5 & Germany \\
\hline Hidrue et al. (2011) & 1 & 1 & USA \\
\hline Horne et al. (2005) & 2 & 2 & Canada \\
\hline Mau et al. (2008) & 1 & 1 & Canada \\
\hline Ziegler (2012) & 4 & 4 & Germany \\
\hline N & & 34 & \\
\hline
\end{tabular}

From 14 studies (see Table 6 ) a total of 34 WTP ratios were extracted. These covered 7 different fuel types LPG ( $n=3)$, CNG ( $n=2)$, Meth $(n=1)$, Biofuel $(n=1)$ FCEV $(n=3)$, PHEV $(n=6)$, Electric $(n=7)$ with 3 studies measuring AFV as a generic fuel type. Five countries were represented in the data, Germany $(n=14)$, South Korea $(n=3)$, Canada $(n=6)$, USA $(n=9)$ and Norway $(n=2)$.

Although mean WTP ratios vary across fuel types (Table 7) small sample sizes restrict granularity at this level and we therefore aggregate to AFVs.

Table 7 - Risk premium values across fuel types

\begin{tabular}{|lrrrrrrrrr|}
\hline & \multicolumn{2}{c}{ AFV } & LPG & \multicolumn{2}{c}{ CNG } & \multicolumn{2}{c}{ Meth } & Bio & \multicolumn{2}{c}{ FCEV } & PHEV & Elec \\
Mean WTP/fuel type & $\mathbf{3 6 3 3}$ & $\mathbf{6 0 6 4}$ & $\mathbf{1 6 2 5}$ & $\mathbf{4 6 8 0}$ & $\mathbf{9 8 0}$ & $\mathbf{5 3 6 0}$ & $\mathbf{4 3 9 9}$ & $\mathbf{5 1 2 5}$ \\
Std Dev & $\mathbf{1 1 1 3}$ & $\mathbf{9 0 6}$ & $\mathbf{7 4}$ & & & & $\mathbf{8 4 6 1}$ & $\mathbf{3 6 2 2}$ & $\mathbf{3 6 8 3}$ \\
Sample Size & 3 & 3 & 2 & 1 & 1 & 3 & 6 & 7 \\
\hline
\end{tabular}


A total of 8 WTP ratios were excluded as extreme outliers ( $\geq$ US\$ 201020,000 ) leaving a total sample size of 26. This final sample follows a normal distribution, confirmed by a Shapiro Wilk W test for normality (W=0.89670 | p>z 0.01322).

Although within this smaller sample it was not possible to reliably distinguish between alternative fuel technologies, some regional variation is possible. To ensure robust measurement we group WTP ratios to represent three main regions (1) Global $(n=26),(2)$ USA/Canada $(n=14)$ and (3) Europe $(n=9)$.

In line with Rogers (2003) we identify initial risk premium values for all five adopter categories within each region by calculating from each distribution of WTP ratios point estimates for the mean WTP $(\bar{x} W T P)$ and standard deviation WTP $(\bar{\sigma} W T P)$. Using Rogers (2003) adoption propensity curve we calculate initial risk premium values for $\mathrm{IN}=\bar{x}-3 \bar{\sigma}$ (mid point of $\bar{x}-4 \bar{\sigma}$ to $\bar{x}-2 \bar{\sigma}$ ), EA $=\bar{x}-$ $1.5 \bar{\sigma}$ (midpoint $\bar{x}-2 \bar{\sigma}$ to $\bar{x}-1 \bar{\sigma}$ ), $\mathrm{EM}=\bar{x}-.5 \bar{\sigma}$ (midpoint $\bar{x}-1 \bar{\sigma}$ to $\bar{x}$ ), $\mathrm{LM}=$ $\bar{x}+.5$ (midpoint $\bar{x}$ to $\bar{x}+1 \bar{\sigma}$ ) and $\mathrm{LG}=\bar{x}+2.5 \bar{\sigma}$ (midpoint $\bar{x}+1 \bar{\sigma}$ to $\bar{x}+4 \bar{\sigma}$ ).

Table 8 - Initial risk premium values for IN, EA, EM, LM and LG (WTP AFV).

\begin{tabular}{|l|l|l|l|}
\hline & Global & USA/Canada & Europe \\
\hline All consumers & US\$ ppp 2010 & US\$ ppp 2010 & US\$ ppp 2010 \\
\hline $\mathrm{N}$ & 26 & 14 & 9 \\
\hline Mean & 4475 & 3483 & 6039 \\
\hline Standard deviation & 3676 & 2566 & 5037 \\
\hline Consumer segments & & -4217 & \\
\hline Innovators (IN) & -6553 & -367 & -9073 \\
\hline Early Adopters (EA) & -1039 & & -1517 \\
\hline Early Adopters (IN\&EA) & & 2199 & \\
\hline Early Majority (EM) & 2637 & 4766 & 3521 \\
\hline Late Majority (LM) & 6313 & 9899 & 8558 \\
\hline Laggards (LG) & 13665 & 18633 \\
\hline
\end{tabular}

In terms of regional variation consumers in USA/Canada are on average least risk averse with a mean WTP for AFV of US\$3483 (Table 10). In all three regions IN and EA are receptive to risk (represented by a negative risk premium). It should be noted that a positive WTP indicates a negative risk premium, i. e., risk averse; conversely, a negative WTP is a positive risk premium, i. e. , receptiveness to risk. By design, LM and LG have a higher risk premium so more strongly negative WTP than EM, and the same for EM compared to EA.

In our model formulation we use only four adopters groups. A single risk premium value for IN\&EA is calculated by extrapolating from a linear decline for these two groups individually over 3 standard deviations to zero. To calculate a single initial risk premium value we plot a linear decline for both groups. 


\section{Supplementary information C - Calculation of regional variation in social influence effect sizes}

We illustrate the calculation of country specific effect sizes for social influence using the linear regression equation $y=-0.428 x+0.4497$ (where $y=$ social influence effect size and $x=$ score on pragmatic versus normative scale $\left.\left(R^{2}=0.82\right)\right)$. This equation was derived from a meta-regression model which tested the moderating effects of national culture on social influence measured in 11 different countries. For a full description of this please see Pettifor et al (2017).

To distinguish between countries we use one of (Hofstede, 2001) five cultural dimensions. These dimensions consist of are 'power distance' (the degree to which inequalities are broadly acceptable), 'individuality/collectivism' (the extent to which people seek distinctive individual identity compared to belonging to groups), 'uncertainty avoidance' (the extent to which people feel threatened by unknown situations), 'masculinity/femininity' (masculine cultures are strongly goal orientated compared to feminine cultures which prioritise quality of life) and 'pragmatic/normative' (extent to which people are willing to adapt to changing social conditions compared to maintaining current traditions and holding onto the status quo).

The meta-analysis returned a significant average effect size of beta $=0.241(95 \% \mathrm{Cl}[0.157,0.322]$, $Z=5.505,|p| \leq 0.000)$. This average effect size was based on all studies and countries. However, further testing found that the average effect size was moderated by a country's cultural values, measured by a widely-used scale from 'pragmatic' to 'normative' (Minkov and Hofstede, 2012). This scale quantifies differences between countries' receptiveness to social influence, using data from the World Values Survey. Social influences are stronger for countries at the normative end of the scale (towards zero). In these countries dominant culture is concerned with reinforcing current ways of doing things, established traditions and routines. People prioritise learning from each other as opposed to changing in accordance with new social contexts. Scores on this scale were therefore used to predict social influence effect sizes and confidence intervals for the 11 countries that were sampled within the meta-analysis (Figure 12).

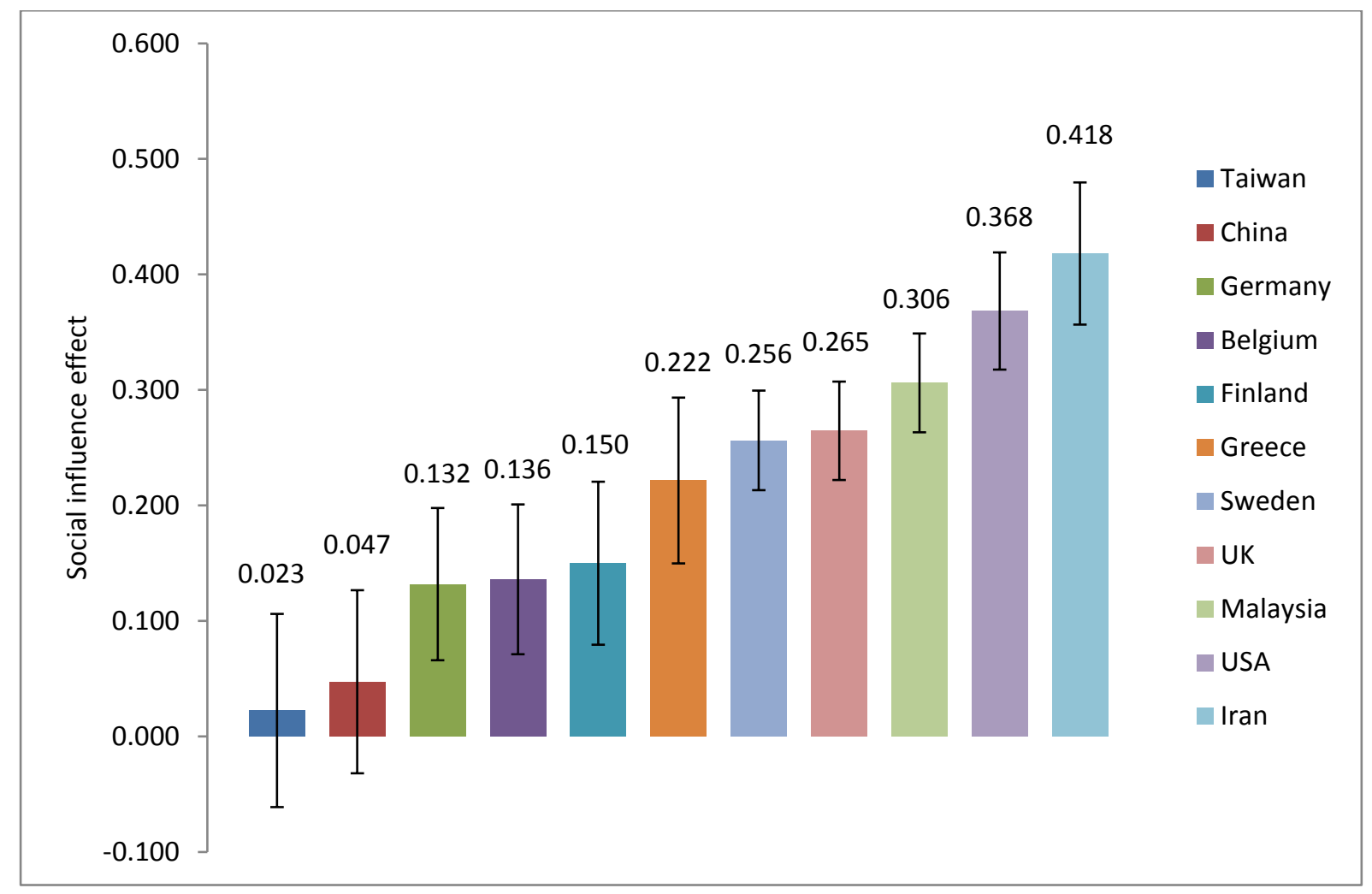


Figure 12 - Social influence effect size for countries included in the meta-analysis, adjusted according to pragmatic versus normative scale (confidence intervals indicated by error bars)

For countries not included in the meta-analysis, their score on this scale (available for over 80 different countries within the 26 IMAGE regions) is used to estimate social influence effect size at country level. This is achieved through plotting the linear association between scores on the pragmatic versus normative scale and predicted social influence effect for the 11 countries sampled in the meta-analysis and using the resultant linear equation to make further out of sample predictions (Figure 13).

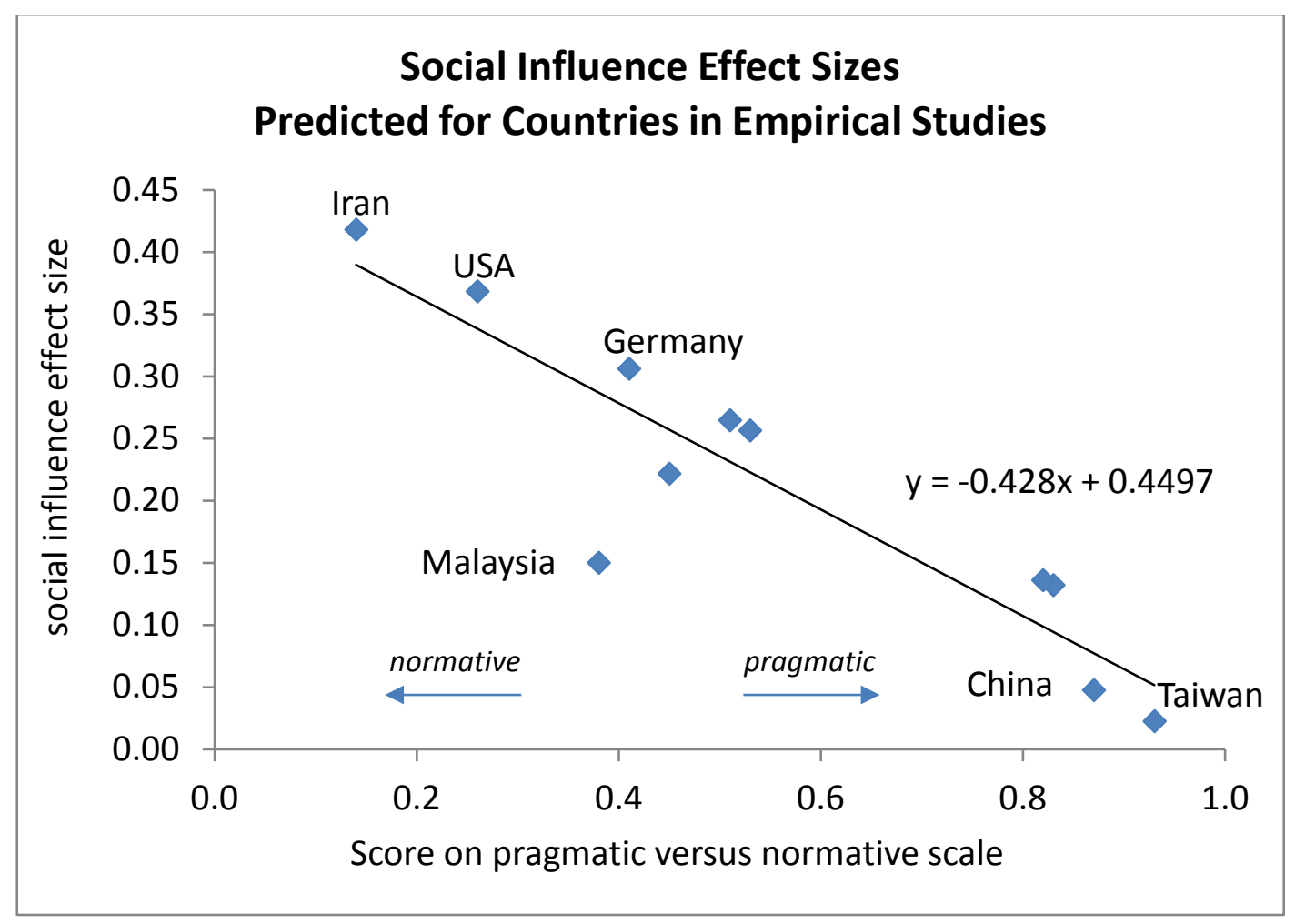

Figure 13 -Linear association between score on pragmatic versus normative scale and social influence effect size for countries sampled within meta-analysis

Countries are then aggregated into model regions again by weighting the social influence effect size according to GDP. If scores on the pragmatic-normative scale are not available for certain countries, social influence effect sizes are calculated from the linear association between country GDP and social influence effect size, taking values from a minimum number of $10 \%$ of countries within a particular region (Table 9). GDP was selected as the most appropriate mechanism through which to weight countries because the pragmatic - $v$ - normative scale also differentiates between long term and short term investment cultures. For example highly pragmatic cultures have a longer term orientation towards growth and investment. The weighting according to GDP has a modest effect, countries in a particular geographical region have similar scores on the pragmatic-v-normative scale.

Table 9 - Calculation of social influence effect size for representative countries within regions

\section{Approach}

(a) Included in meta-analysis based on empirical studies, hence directly estimated from score on pragmatic versus normative scale.
Countries affected

Taiwan, China, Germany, Belgium, Sweden, UK, Greece, Malaysia, Finland, USA, Iran. 
(b) Scores available on pragmatic versus normative scale. Extrapolation from linear regression (from countries included in (a)):

$y=-0.428 x+0.4497$

(where $y=$ social influence effect size and $x=$ score on pragmatic versus normative scale $\left.\left(R^{2}=0.82\right)\right)$.

(c) Scores not available on pragmatic versus normative scale. Estimation from GDP using linear regression between social influence effect size and GDP (from countries included in (a) and (b)):

$y=0.000002 x+0.3124$

(where $y=$ social influence effect size and $x=$ country GDP per capita (US\$ 2010) $\left.\left(R^{2}=0.1214\right)\right)$.
Mexico, Dominican Republic, El Salvador, Trinidad and Tobago, Bolivia, Chile, Columbia, Peru, Uruguay, Venezuela, Libya, Morocco, Egypt, Cape Verde, Ghana, Nigeria, Austria, Denmark, France, Iceland, Ireland, Italy, Luxembourg, Malta, Netherlands, Norway, Portugal, Spain, Switzerland, Albania, Bulgaria, Croatia, Czech Republic, Estonia, Hungary, Latvia, Lithuania, Poland, Romania, Slovak Republic, Slovenia, Serbia and Montenegro, Russian Federation, Israel, Jordan, Lebanon, Saudi Arabia, Syrian Arab Rep, Hong Kong, Philippines, Singapore, Vietnam, Thailand, Indonesia, Australia, New Zealand, Bangladesh, Sri Lanka, Pakistan, Botswana, Mozambique, Namibia, Tanzania, Zambia.

Ethiopia, Kenya, Madagascar, Mauritius, Rwanda, Sudan, Uganda, Belarus, Moldova, Ukraine, Kazakhstan, Tajikistan, Turkmenistan, Uzbekistan, Korea Rep.

This results in the following values for social influence effect size within each region (Table 10)

Table 10 - Social influence effect sizes for each IMAGE model region

\begin{tabular}{|l|l|l|l|}
\hline IMAGE Region & $\begin{array}{l}\text { Social } \\
\text { Influence } \\
\text { Effect Size }\end{array}$ & & \\
\hline Canada & 0.296 & Ukraine Region & 0.321 \\
\hline USA & 0.368 & Central Asia & 0.326 \\
\hline Mexico & 0.347 & Russia Region & 0.103 \\
\hline Central America & 0.390 & Middle East & 0.324 \\
\hline Brazil & 0.261 & India & 0.231 \\
\hline Rest of South America & 0.354 & Korea region & 0.357 \\
\hline Northern Africa & 0.372 & China Region & 0.108 \\
\hline Western Africa & 0.405 & South Eastern Asia & 0.202 \\
\hline Eastern Africa & 0.340 & Indonesia region & 0.184 \\
\hline South Africa & 0.320 & Japan & 0.073 \\
\hline Western Europe & 0.222 & Oceania & 0.337 \\
\hline Central Europe & 0.176 & Rest of South Asia & 0.249 \\
\hline Turkey & 0.253 & Rest of Southern Africa & 0.336 \\
\hline
\end{tabular}




\section{Supplementary information D - Calculation of decline function for endogenous model formulation and input equations}

We describe the endogenous risk premium decline function used for the IMAGE model. In summary we apply the regression equation from the meta-analysis $(+1 \bar{\sigma} M K=0.241 * \bar{\sigma} R P)$ to derive the rate at which risk premium declines as a result of market share growth. Because this is an endogenous relationship (and we cannot hardcode a decline as in the case of the exogenous formulation) we derive a stepwise linear function which adjusts the linear regression equation to reflect five 'steps' of market share growth -0 to $2.5 \%, 2.5 \%$ to $16 \%, 60 \%$ to $50 \%, 50 \%$ to $84 \%$ and $84 \%$ to $100 \%$ (these correspond to adopter types from Rogers diffusion of innovations). For example for market share growth between $2.5 \%$ and just below $16 \%$ the equation is $+3 \bar{\sigma} M K=3 * 0.241 *$ $\bar{\sigma} R P$.

In our endogenous model formulation market share growth is divided into standard deviations using the normal distribution curve. This standardises the axis for market share growth in terms of numbers of standard deviations such that $2.5 \% \mathrm{MK}=2 \bar{\sigma} M K, 16 \% \mathrm{MK}=3 \bar{\sigma} M K, 50 \% \mathrm{MK}=4 \bar{\sigma} M K$, $84 \% \mathrm{MK}=5 \bar{\sigma} M K$ and $100 \% \mathrm{MK}=6 \bar{\sigma} M K$.

We use this to calculate a decline function between these values of market share which is conditional on $\bar{\sigma} M K$ ie. ,

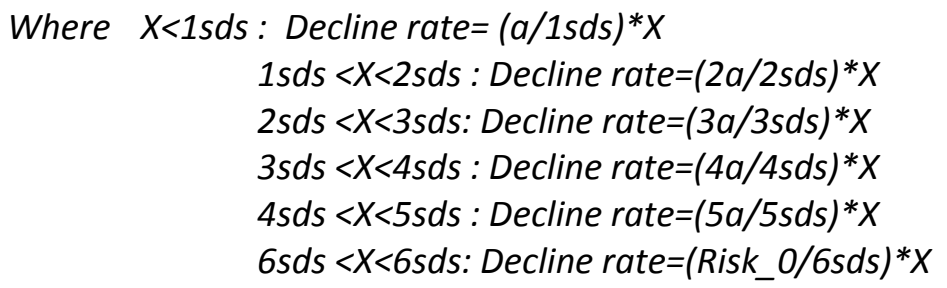

Where $a=$ social influence effect size ${ }^{*} \bar{\sigma} R P$

And $X=$ market share

Table 11 - Decline rates incorporated into endogenous formulation

\begin{tabular}{|l|l|l|}
\hline & EA & EM, LM, LG \\
\hline $\bar{\sigma} R P$ (global) & 3.676 & 3.676 \\
\hline Global decline rate $(0.24 * \bar{\sigma} R P)$ & 0.886 & 0.886 \\
\hline & & \\
\hline $\bar{\sigma} R P($ USA) & 2.566 & 2.566 \\
\hline USA decline rate $(0.36 * \bar{\sigma} R P)$ & 0.92 & 0.92 \\
\hline & & \\
\hline $\bar{\sigma} R P($ WEU) & 5.037 & 5.037 \\
\hline Global decline rate $\left(0.22^{*} \bar{\sigma} R P\right)$ & 1.11 & 1.11 \\
\hline
\end{tabular}




\section{Supplementary Information E - Calculation of decline function for exogenous model formulation}

The decline in risk premium is conceptualised as being the result of social influence effects, i.e., early adoption reduces the perceived risk of AFVs among later adopting market segments. Although social influence effects are not modelled directly in MESSAGE the number of AFVs are endogenously generated by MESSAGE and expressed as absolute numbers (millions of vehicles) or market share (\% of all vehicles). Using increasing market share as a proxy for social influence effects creates an endogeneity problem as market share is both a model output and a basis for the social influence input. A work around is to use a base model run (without social influence effect) to estimate changing market share over time, and then use this time series to exogenously estimate changing risk premium over time. Taking this approach enables market share to be directly converted into a distribution over time. Market share is taken directly from MESSAGE base model output (Table 12). Output is from a stringent climate mitigation scenario (reaching $~ 500$ ppm CO2-eq in 2100) constrained by a global $\mathrm{CO} 2$-eq budget and with optimal assumptions that mitigation takes place where and when it is most cost-effective (including across sectors and regions). The scenario includes risk premiums (intangible costs) for three consumer segments (adopter types) but no social influence effects, i.e., risk premiums remain constant through time at their initial values (EA: -2433 \$/veh; EM: 725 \$/veh; LM: 3827 \$/veh). Note that the 'NAM' (North America) region in MESSAGE is reflective of the USA.

Table 12 - Growth in share of AFVs over time, all regions and NAM

\begin{tabular}{|l|l|l|l|l|l|l|l|l|l|l|l|}
\hline \multicolumn{1}{|l|}{ Growth in market share } \\
\hline & 1990 & 2000 & 2005 & 2010 & 2020 & 2030 & 2040 & 2050 & 2060 & 2070 & 2080 \\
\hline All Regions & 0.00 & 0.00 & 0.00 & 0.00 & 0.00 & 0.07 & 0.15 & 0.32 & 0.62 & 0.89 & 1.00 \\
\hline NAM & 0.00 & 0.00 & 0.00 & 0.00 & 0.00 & 0.08 & 0.21 & 0.60 & 0.98 & 1.00 & 1.00 \\
\hline
\end{tabular}

To derive an input/independent ' $X$ ' variable which is consistent with the approach taken in the metaanalysis, mean and standard deviations are required from these distributions. Mean market share is a straight forward calculation using the mid-point of the diffusion curve, i. e. , when the $50 \%$ percentile has adopted (assuming a logistic curve function). The distribution of market share for which a standard deviation can be estimated is then conceptualised as the first derivative of the diffusion curve analogous to Rogers' adoption propensity curve. In other words, cumulative adoption over time through Rogers' five adopter categories gives rise to the observed diffusion curve showing the increasing market share of AFVs. Using a simplifying assumption that the Rogers' curve is truncated $+/-4$ standard deviations from the mean (similar to the approach taken earlier for estimating WTPs) we can infer that innovators (I) adopt between -4 and -2 standard deviations below the mean, EA adopt between -2 and -1 standard deviations, EM adopt between -1 and 0 standard deviations, and so on. The diffusion curve expressed as changing market share over time can be used to estimate the value of a standard deviation in terms of numbers of years. Where diffusion to the mean occurs over a longer duration, there is a subsequently higher standard deviation, and vice versa. 


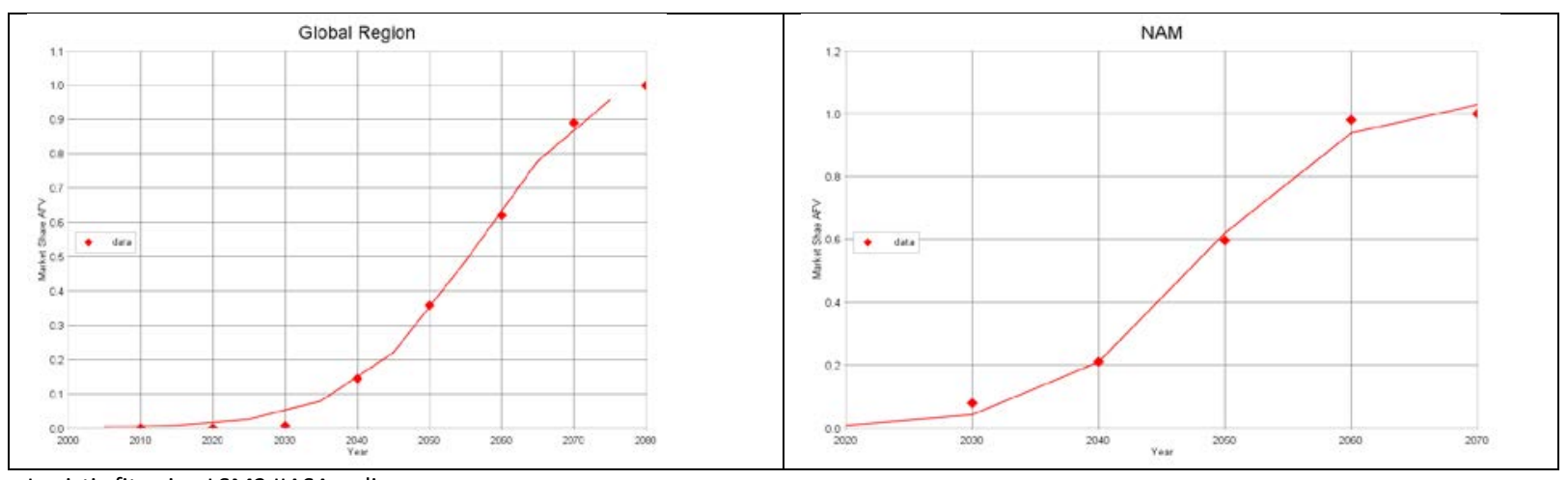

Logistic fit using LSM2 IIASA online

Figure 14 - Logistic fit to market share data points, all regions (global) and NAM.

For both distributions, logistic curves are fitted (Figure 14) and mean market share identified. The duration of diffusion to this midpoint estimate then enables a calculation of standard deviation (in years) which converts the market share data points into time series data (Table 13).

Table 13 - Conversion of market share data from MESSAGE to time series

\begin{tabular}{|c|c|c|c|c|c|c|}
\hline & $\begin{array}{l}\text { AFV } \\
\text { diffusion } \\
\text { begins (yrs) }\end{array}$ & $\begin{array}{l}\text { AFV } \\
\text { saturation } \\
\text { point } \\
\text { (yrs) } \\
\end{array}$ & $\begin{array}{l}\text { Duration } \\
\text { (yrs) }\end{array}$ & $\begin{array}{l}50 \% \text { market } \\
\text { share (mean) } \\
\text { (yrs) }\end{array}$ & $\begin{array}{l}\text { Duration to } \\
50 \% \text { market } \\
\text { share } \\
\text { (yrs) }\end{array}$ & $\begin{array}{l}1 \mathrm{Sd} \\
\text { market } \\
\text { share } \\
\text { (yrs) } \\
\end{array}$ \\
\hline & (a) & (b) & (b)-(a) & (d) & (d)-(a) & (d)-(a)/4 \\
\hline All regions & 2005 & 2080 & 75 & 2056 & 51 & 12.75 \\
\hline NAM & 2020 & 2070 & 50 & 2048 & 28 & 7 \\
\hline
\end{tabular}

According to MESSAGE base run take-up of AFVs occurs more quickly in NAM, i.e., standard deviation in market share $=7$ years compared to 12.75 years for all regions. This is consistent with NAM being an innovator region into which advanced technologies diffuse more rapidly than the global average. The calculations for $1 \mathrm{sd}$ market share (time) are used as a basis to scale the ' $X$ ' axis (in yearly/10 year increments), and this is multiplied by social influence effect size $(\bar{x}=0.24)$ to calculate the rate of decline of risk premium. This is in accordance with the original findings from the meta-analysis i. e. , a 1 standard deviation increase in social influence effect results in a 0.241 increase in the propensity to purchase a particular type of vehicle.

Table 14 shows the resultant linear decline functions for each region which are used to hardcode decline rates into MESSAGE.

Table 14 - Linear decline function model inputs per adopter group

\begin{tabular}{|l|l|l|l|l|}
\hline Adopter group & EA & EM & LM & LG \\
\hline Global average & $\mathrm{Y}=0.116 \mathrm{x}-3.5333$ & $\mathrm{Y}=-0.069 \mathrm{x}+2.637$ & $\mathrm{Y}=-0.069 \mathrm{x}+6.313$ & $\mathrm{Y}=-0.069 \mathrm{x}+13.665$ \\
\hline North America & $\mathrm{Y}=0.063 \mathrm{x}-1.571$ & $\mathrm{Y}=-0.1372 \mathrm{x}+2.199$ & $\mathrm{Y}=-0.1331 \mathrm{x}+4766$ & $\mathrm{Y}=-0.1336 \mathrm{x}+9.899$ \\
\hline
\end{tabular}

We note that there is likely to be some inconsistency in the modelling results due to the manner in which the hardcoded decline rates are used as a 'new' baseline for no social influence effect (NoSI scenario). Table 15 illustrates this difference in terms of the scaling used for the exogenous decline function. What we see is the association between risk premium decline and market share strengthens (speeds up) under NoSI scenario compared to the original baseline run by 1 year (from 12.75 years per $1 \mathrm{sd}$ market share to 11.75 years). Whilst this results in some small changes to total 
time taken to reach $50 \%$ market share of AFVs between the base run and NoSI scenario (4 years) we anticipate a minimal effect on the overall diffusion curves.

Table 15 - Sensitivity test, parameters within the exogenous decline function

\begin{tabular}{|c|c|c|c|c|c|c|}
\hline & $\begin{array}{l}\text { AFV } \\
\text { diffusion } \\
\text { begins (yrs) }\end{array}$ & $\begin{array}{l}\text { AFV } \\
\text { saturation } \\
\text { point } \\
\text { (yrs) } \\
\end{array}$ & $\begin{array}{l}\text { Duration } \\
\text { (yrs) }\end{array}$ & $\begin{array}{l}50 \% \text { market } \\
\text { share (mean) } \\
\text { (yrs) }\end{array}$ & $\begin{array}{l}\text { Duration to } \\
50 \% \text { market } \\
\text { share } \\
\text { (yrs) }\end{array}$ & $\begin{array}{l}1 \text { Sd } \\
\text { market } \\
\text { share } \\
\text { (yrs) } \\
\end{array}$ \\
\hline & (a) & (b) & (b)-(a) & (d) & (d)-(a) & (d)-(a)/4 \\
\hline Base run & 2005 & 2080 & 75 & 2056 & 51 & 12.75 \\
\hline NoSI & 2010 & 2100 & 90 & 2057 & 47 & 11.75 \\
\hline GlobalSI & 2010 & 2100 & 90 & 2054 & 44 & 11 \\
\hline RegSI & 2010 & 2100 & 90 & 2049 & 39 & 9.75 \\
\hline
\end{tabular}




\section{Supplementary information F - Technology groupings within models}

Table 16 - Full technology groupings within models

\begin{tabular}{|l|l|l|l|}
\hline & $\begin{array}{l}\text { Conventional Fuel } \\
\text { types (CV types) }\end{array}$ & $\begin{array}{l}\text { Alternative fuel vehicle types } \\
\text { (AFV types) }\end{array}$ & Electric vehicle types (EV types) \\
\hline IMAGE & Fossil ICE, Fossil HEV & $\begin{array}{l}\text { Biofuel ICE, Hydrogen ICE, } \\
\text { Natural Gas HEV, Biofuel HEV, } \\
\text { Hydrogen HEV, Hydrogen FCV }\end{array}$ & $\begin{array}{l}\text { Battery-Electric, Fossil PHEV, } \\
\text { Biofuel PHEV }\end{array}$ \\
\hline MESSAGE & Fossil ICE & $\begin{array}{l}\text { Fossil HEV, Natural Gas ICE, } \\
\text { Natural Gas HEV, Biofuel HEV, } \\
\text { Fossil Synfuel ICE, Fossil Synfuel } \\
\text { HEV, Hydrogen FCV }\end{array}$ & $\begin{array}{l}\text { Battery-Electric, Fossil/Biofuel } \\
\text { PHEV }\end{array}$ \\
& & \\
\hline
\end{tabular}




\section{Supplementary information G - Results of sensitivity run StrongSI scenario}

In the StrongSI scenario we test the effect on global vehicle deployment of increasing the social influence effect size from 0.241 (the global average) to 0.322 (the upper $95 \%$ confidence interval) based on our meta-analysis (Supplementary Information A).

Our earlier analysis showed that stronger social influence effects speeds up risk premium decline in all adopter groups and strengthens interdependencies between earlier and later adopters. This results in greater overall differentiation between adopter groups, and a fuller transition to BEVs among later adopters. This basic pattern also occurs in the StrongSI scenario, but across all model regions.

Table 17 shows a breakdown of vehicle deployment in 2050 and 2100 contrasting the GlobalSI and StrongSI scenarios. Increasing the strength of the social influence effect slightly stimulates deployment of AFVs overall. By $210061 \%$ of all passenger vehicle-kilometres (vkm) are travelled in AFVs, compared to $52 \%$ in the GlobalSI scenario (Table 17). Within this slightly fuller transition to all types of AFV, social influence effects also result in slightly more differentiation with a higher share of PHEVs and BEVs in 2100 (52\% compared to $43 \%$ in GlobalSI).

Table 17 - Total vehicle deployment (\% vkm) in the IMAGE model, comparing GlobalSI scenario and StrongSI scenario

\begin{tabular}{|c|c|c|c|c|c|c|c|c|}
\hline \multirow[b]{2}{*}{ \% vkm globally } & \multicolumn{2}{|c|}{ NoSI Scenario } & \multicolumn{2}{|c|}{ GlobaISI Scenario } & \multicolumn{2}{|c|}{$\begin{array}{r}\text { RegionalSI } \\
\text { Scenario }\end{array}$} & \multicolumn{2}{|c|}{ StrongSI Scenario } \\
\hline & 2050 & 2100 & 2050 & 2100 & 2050 & 2100 & 2050 & 2010 \\
\hline CV & 0.85 & 0.78 & 0.86 & 0.48 & 0.86 & 0.43 & 0.86 & 0.39 \\
\hline AFV (all types) & 0.15 & 0.22 & 0.14 & 0.52 & 0.14 & 0.57 & 0.14 & 0.61 \\
\hline of which ... & & & & & & & & \\
\hline other AFV & 0.15 & 0.00 & 0.40 & 0.15 & 0.40 & 0.1 & 0.40 & 0.13 \\
\hline PHEV & 0.85 & 0.08 & 0.60 & 0.01 & 0.60 & 0.02 & 0.60 & 0.02 \\
\hline BEV & 0.00 & 0.92 & 0.00 & 0.83 & 0.00 & 0.84 & 0.00 & 0.85 \\
\hline
\end{tabular}

These overall effects are small and it is likely that our use of the upper bound of social influence effects from meta-analysis of empirical studies is conservative, as these empirical studies included few contexts in which AFVs were being strongly promoted. 